\title{
GENERAL EXISTENCE PRINCIPLES FOR NONLOCAL BOUNDARY VALUE PROBLEMS WITH $\phi$-LAPLACIAN AND THEIR APPLICATIONS
}

\author{
RAVI P. AGARWAL, DONAL O’REGAN, AND SVATOSLAV STANĚK
}

Received 1 April 2005; Accepted 12 May 2005

The paper presents general existence principles which can be used for a large class of nonlocal boundary value problems of the form $\left(\phi\left(x^{\prime}\right)\right)^{\prime}=f_{1}\left(t, x, x^{\prime}\right)+f_{2}\left(t, x, x^{\prime}\right) F_{1} x+$ $f_{3}\left(t, x, x^{\prime}\right) F_{2} x, \alpha(x)=0, \beta(x)=0$, where $f_{j}$ satisfy local Carathéodory conditions on some $[0, T] \times \mathscr{D}_{j} \subset \mathbb{R}^{2}, f_{j}$ are either regular or have singularities in their phase variables $(j=$ $1,2,3), F_{i}: C^{1}[0, T] \rightarrow C^{0}[0, T](i=1,2)$, and $\alpha, \beta: C^{1}[0, T] \rightarrow \mathbb{R}$ are continuous. The proofs are based on the Leray-Schauder degree theory and use regularization and sequential techniques. Applications of general existence principles to singular BVPs are given.

Copyright (C) 2006 Ravi P. Agarwal et al. This is an open access article distributed under the Creative Commons Attribution License, which permits unrestricted use, distribution, and reproduction in any medium, provided the original work is properly cited.

\section{Introduction}

Let $T>0$. As usual, $C^{j}[0, T]$ and $\mathrm{AC}^{j}[0, T](j=0,1)$ denote the set of functions having the $j$ th derivative continuous and absolutely continuous on $[0, T]$, respectively. $L_{1}[0, T]$ is the set of Lebesgue integrable functions on $[0, T]$. In what follows, $C^{0}[0, T]$ and $L_{1}[0, T]$ are equipped with the norms

$$
\|x\|=\max \{|x(t)|: 0 \leq t \leq T\}, \quad\|x\|_{L}=\int_{0}^{T}|x(t)| d t,
$$

respectively.

Assume that $G \subset \mathbb{R}^{2}$. $\operatorname{Car}([0, T] \times G)$ stands for the set of functions $f:[0, T] \times G \rightarrow$ $\mathbb{R}$ satisfying local Carathéodory conditions on $[0, T] \times G$, that is: $(\mathrm{j})$ for each $(x, y) \in$ $G$, the function $f(\cdot, x, y):[0, T] \rightarrow \mathbb{R}$ is measurable; (ji) for a.e. $t \in[0, T]$, the function $f(t, \cdot, \cdot): G \rightarrow \mathbb{R}$ is continuous; (jij) for each compact set $K \subset G$, $\sup \{|f(t, x, y)|$ : $(x, y) \in K\} \in L_{1}[0, T]$. For any measurable $M \subset \mathbb{R}, \mu(M)$ denotes the Lebesgue measure of $M$. 
2 General existence principles for nonlocal BVPs

We will denote by $\mathscr{H}$ the set of operators $F: C^{1}[0, T] \rightarrow C^{0}[0, T]$ which are

(a) continuous and

(b) bounded, that is, for any $r>0$,

$$
\sup \left\{\|F x\|: x \in C^{1}[0, T],\|x\|+\left\|x^{\prime}\right\| \leq r\right\}<\infty \text {. }
$$

Finally, let $\mathscr{A}$ denote the set of functionals $\alpha: C^{1}[0, T] \rightarrow \mathbb{R}$ which are

(a) continuous and

(b) bounded, that is, $\alpha(\Omega)$ is bounded (in $\mathbb{R}$ ) for any bounded $\Omega \subset C^{1}[0, T]$.

We study singular nonlocal boundary value problems (BVPs) of the type

$$
\begin{aligned}
\left(\phi\left(x^{\prime}(t)\right)\right)^{\prime}= & f_{1}\left(t, x(t), x^{\prime}(t)\right)+f_{2}\left(t, x(t), x^{\prime}(t)\right)\left(F_{1} x\right)(t) \\
+ & f_{3}\left(t, x(t), x^{\prime}(t)\right)\left(F_{2} x\right)(t) \\
& \alpha(x)=0, \quad \beta(x)=0,
\end{aligned}
$$

where $\phi$ is an increasing homeomorphism from $\mathbb{R}$ onto $\mathbb{R}, f_{j} \in \operatorname{Car}\left([0, T] \times \mathscr{D}^{j}\right)$, the sets $\mathscr{D}^{j}=\mathscr{D}_{1}^{j} \times \mathscr{D}_{2}^{j} \subset \mathbb{R}^{2}$ are not necessarily closed, $f_{j}$ have singularities in their phase variables on the boundary $\partial \mathscr{D}^{j}$ of $\mathscr{D}^{j}(j=1,2,3), F_{i} \in \mathscr{H}^{\prime}(i=1,2)$ and $\alpha, \beta \in \mathscr{A}$.

Let $j \in\{1,2,3\}$. We say that $f_{j}$ has a singularity on $\partial \mathscr{D}^{j}$ in its phase variable $x_{i}(i=1,2)$ if there exists $a_{i, j} \in \partial \mathscr{D}_{i}^{j}$ such that

$$
\limsup _{x_{i} \rightarrow a_{i, j}, x_{i} \in \mathscr{D}_{i}^{j}}\left|f_{j}\left(t, x_{1}, x_{2}\right)\right|=\infty
$$

for a.e. $t \in[0, T]$ and all $x_{3-i} \in D_{3-i}^{j}$.

A function $x \in C^{1}[0, T]$ is said to be a solution of the BVP (1.3), (1.4) if $\phi\left(x^{\prime}\right) \in$ $\mathrm{AC}[0, T], x$ satisfies the boundary conditions (1.4) and (1.3) holds for almost all $t \in$ $[0, T]$.

Nonlocal BVPs for second-order differential equations with nonlinear left-hand sides and having singularities in their phase variables were studied in $[1,2,5,10-12]$. In [1] the differential equation $(g(x))^{\prime \prime}=f\left(t, x,(g(x))^{\prime}\right)$ is discussed together with the nonlocal boundary conditions $x(0)=x(T), \min \{x(t): 0 \leq t \leq T\}=0$. In [2] the authors present conditions guaranteeing that the BVP $\left(\phi\left(x^{\prime}\right)\right)^{\prime}=\mu f\left(t, x, x^{\prime}\right), x(0)=0=x(T)$, $\max \{x(t): 0 \leq t \leq T\}=A$ has for any $A>0$ a positive solution with a positive value of the parameter $\mu$. The existence of a solution of $\left(\phi\left(x^{\prime}\right)\right)^{\prime}=f\left(t, x, x^{\prime}\right)$ satisfying $x(0)=x(T)$, $\max \{x(t): 0 \leq t \leq T\}=A$ is considered in [11], satisfying $x(0)=x(T)=-\gamma \min \{x(t)$ : $0 \leq t \leq T\}(\gamma \in(0, \infty))$ in [10] and satisfying $\min \{x(t): 0 \leq t \leq T\}=0, \chi\left(x^{\prime}\right)=0$ where $\chi$ is a continuous functional in [12]. Existence results for functional-differential equations with nonlinear functional left-hand sides and nonlocal functional boundary conditions are also presented in [5].

The aim of this paper is to present general existence principles for solving regular and singular nonlocal BVPs for second-order functional-differential equations with $\phi$ Laplacian and to give applications of these general principles. The general existence principle for regular nonlocal BVPs can be used either for solving regular BVPs or in the case 
of singular BVPs for solving a sequence of auxiliary regular BVPs obtaining by regularization and sequential techniques. We note that our general existence principle for singular nonlocal BVPs is related to that given in [9] for singular BVPs

$$
x^{(n)}(t)=h\left(t, x(t), \ldots, x^{(n-1)}(t)\right), \quad x \in \mathscr{Y}
$$

where $h$ has singularities in all its phase variables and $\mathscr{Y}$ is a closed subset of $C^{n-1}[0, T]$.

To obtain a solution of the BVP (1.3), (1.4), we use regularization and sequential techniques. To use these techniques we consider a sequence of regular functional-differential equations

$$
\begin{aligned}
\left(\phi\left(x^{\prime}(t)\right)\right)^{\prime}= & f_{1, n}\left(t, x(t), x^{\prime}(t)\right)+f_{2, n}\left(t, x(t), x^{\prime}(t)\right)\left(F_{1} x\right)(t) \\
& +f_{3, n}\left(t, x(t), x^{\prime}(t)\right)\left(F_{2} x\right)(t),
\end{aligned}
$$

where $f_{j, n} \in \operatorname{Car}\left([0, T] \times \mathbb{R}^{2}\right), n \in \mathbb{N}, j=1,2,3$. If $x_{n}$ is a solution of the BVP (1.7), (1.4), then a solution of the BVP (1.3), (1.4) is obtained as the limit (in $C^{1}[0, T]$ ) of a subsequence of $\left\{x_{n}\right\}$. In limiting processes one usually uses the Lebesgue dominated convergence theorem. Note that in our case with general nonlocal boundary conditions (1.4), we often cannot find a Lebesgue integrable majorant function for the auxiliary sequence of regular functions connected to the BVP (1.3), (1.4). In such a case our limiting processes are based on Vitali's convergence theorem, where the assumption about the existence of a Lebesgue integrable majorant function is replaced by a more general assumption about the uniform integrability.

A collection $\mathscr{V} \subset L_{1}[0, T]$ is called uniformly integrable (UI) on $[0, T]$ if to given $\varepsilon>0$ there exists $\delta>0$ such that if $\varrho \in \mathscr{V}$ and $\mathcal{M} \subset[0, T], \mu(\mathcal{M})<\delta$, then

$$
\int_{\mathcal{M}}|\varrho(t)| d t<\varepsilon
$$

Theorem 1.1 (Vitali's convergence theorem, [3]). Let $\left\{\varrho_{n}\right\}$ be a sequence in $L_{1}[0, T]$ which convergent to $\varrho$ for a.e. $t \in[0, T]$. Then the following statements are equivalent:

(a) $\varrho \in L_{1}[0, T]$ and $\lim _{n \rightarrow \infty}\left\|\varrho_{n}-\varrho\right\|_{L}=0$,

(b) the sequence $\left\{\varrho_{n}\right\}$ is UI on $[0, T]$.

Remark 1.2. Assumption (b) in Theorem 1.1 is equivalent to the following condition: to given $\varepsilon>0$ there exists $\delta>0$ such that for any at most countable set $\left\{a_{j}, b_{j}\right\}_{j \in \rrbracket}$ of mutually disjoint intervals $\left(a_{j}, b_{j}\right) \subset(0, T), \sum_{j \in \mathbb{D}}\left(b_{j}-a_{j}\right)<\delta$, we have

$$
\sum_{j \in \rrbracket} \int_{a_{j}}^{b_{j}}\left|\varrho_{n}(t)\right| d t<\varepsilon, \quad n \in \mathbb{N} .
$$

The rest of the paper is organized as follows. In Section 2, we present a general existence principle for regular nonlocal BVP and general existence principles for singular nonlocal BVP. Applications of both principles are given in Sections 3 and 4. Section 3 discusses singular nonlocal BVPs where nonlinearities in the singular differential equations 
4 General existence principles for nonlocal BVPs

are positive. Section 4 is devoted to the study of positive solutions to singular Dirichlet BVPs for functional-differential equations with right-hand sides changing their sign. Results are demonstrated with examples throughout.

\section{General existence principles}

We first denote by $\mathscr{L}$ the set of functionals $F: C^{1}[0, T] \rightarrow L_{1}[0, T]$ which are

(a) continuous and

(b) for each $r>0$,

$$
\sup \left\{|(F x)(t)|: x \in C^{1}[0, T],\|x\|+\left\|x^{\prime}\right\| \leq r\right\} \in L_{1}[0, T]
$$

Notice that for each $f \in \operatorname{Car}\left([0, T] \times \mathbb{R}^{2}\right)$, the operator $F: C^{1}[0, T] \rightarrow L_{1}[0, T]$ with $(F x)(t)=f\left(t, x(t), x^{\prime}(t)\right)$, belongs to the set $\mathscr{L}$.

Consider the regular functional-differential equation

$$
\left(\phi\left(x^{\prime}(t)\right)\right)^{\prime}=(F x)(t)
$$

where $F \in \mathscr{L}$. We give a general existence principle for the BVP (2.2), (1.4).

Theorem 2.1 (general existence principle for regular nonlocal BVP). Let $\phi$ be an increasing homeomorphism from $\mathbb{R}$ onto $\mathbb{R}, F \in \mathscr{L}$ and $\alpha, \beta \in \mathscr{A}$. Suppose there exist positive constants $S_{0}$ and $S_{1}$ such that

$$
\|x\|<S_{0}, \quad\left\|x^{\prime}\right\|<S_{1}
$$

for all solutions $x$ to the BVP

$$
\begin{aligned}
& \left(\phi\left(x^{\prime}(t)\right)\right)^{\prime}=\lambda(F x)(t) \\
& \alpha(x)=0, \quad \beta(x)=0
\end{aligned}
$$

and each $\lambda \in[0,1]$. Also assume there exists positive constants $\Lambda_{0}$ and $\Lambda_{1}$ such that

$$
|A|<\Lambda_{0}, \quad|B|<\Lambda_{1}
$$

for all solutions $(A, B) \in \mathbb{R}^{2}$ of the system

$$
\begin{aligned}
& \alpha(A+B t)-\mu \alpha(-A-B t)=0 \\
& \beta(A+B t)-\mu \beta(-A-B t)=0
\end{aligned}
$$

and each $\mu \in[0,1]$. Then the BVP (2.2), (1.4) has a solution.

Proof. Set

$$
\Omega=\left\{x: x \in C^{1}[0, T],\|x\|<\max \left\{S_{0}, \Lambda_{0}+\Lambda_{1} T\right\},\left\|x^{\prime}\right\|<\max \left\{S_{1}, \Lambda_{1}\right\}\right\} .
$$


Then $\Omega$ is an open, bounded and symmetric with respect to $0 \in C^{1}[0, T]$ subset of the Banach space $C^{1}[0, T]$. Define the operator $\mathscr{P}:[0,1] \times \bar{\Omega} \rightarrow C^{1}[0, T]$ by the formula

$$
\mathscr{P}(\lambda, x)(t)=x(0)+\alpha(x)+\int_{0}^{t} \phi^{-1}\left(\phi\left(x^{\prime}(0)+\beta(x)\right)+\lambda \int_{0}^{s}(F x)(v) d v\right) d s
$$

A standard argument shows that $\mathscr{P}$ is a continuous operator. We claim that $\mathscr{P}([0,1] \times \bar{\Omega})$ is compact in $C^{1}[0, T]$. Indeed, since $\bar{\Omega}$ is bounded in $C^{1}[0, T]$,

$$
|\alpha(x)| \leq r, \quad|\beta(x)| \leq r, \quad|(F x)(t)| \leq \varrho(t)
$$

for a.e. $t \in[0, T]$ and $x \in \bar{\Omega}$, where $r$ is a positive constant and $\varrho \in L_{1}[0, T]$. Then

$$
\begin{aligned}
|\mathscr{P}(\lambda, x)(t)| \leq \max \left\{S_{0}, \Lambda_{0}+\Lambda_{1} T\right\}+r+T \phi^{-1}\left(\phi\left(\max \left\{S_{1}, \Lambda_{1}\right\}+r\right)+\|\varrho\|_{L}\right), \\
\left|\mathscr{P}(\lambda, x)^{\prime}(t)\right| \leq \phi^{-1}\left(\phi\left(\max \left\{S_{1}, \Lambda_{1}\right\}+r\right)+\|\varrho\|_{L}\right), \\
\left|\phi\left[\mathscr{P}(\lambda, x)^{\prime}\left(t_{2}\right)\right]-\phi\left[\mathscr{P}(\lambda, x)^{\prime}\left(t_{1}\right)\right]\right| \leq\left|\int_{t_{1}}^{t_{2}} \varrho(t) d t\right|
\end{aligned}
$$

for $t, t_{1}, t_{2} \in[0, T]$ and $(\lambda, x) \in[0,1] \times \bar{\Omega}$. Hence $\mathscr{P}([0,1] \times \bar{\Omega})$ is bounded in $C^{1}[0, T]$ and $\left\{\phi\left[\mathscr{P}(\lambda, x)^{\prime}(t)\right]\right\}$ is equicontinuous on $[0, T]$. From

$$
\left|\mathscr{P}(\lambda, x)^{\prime}\left(t_{2}\right)-\mathscr{P}(\lambda, x)^{\prime}\left(t_{1}\right)\right|=\left|\phi^{-1}\left[\phi\left(\mathscr{P}(\lambda, x)^{\prime}\left(t_{2}\right)\right)\right]-\phi^{-1}\left[\phi\left(\mathscr{P}(\lambda, x)^{\prime}\left(t_{1}\right)\right)\right]\right|
$$

and $\phi^{-1}$ being an increasing homeomorphism from $\mathbb{R}$ onto $\mathbb{R}$, we deduce that $\{\mathscr{P}(\lambda$, $\left.x)^{\prime}(t)\right\}$ is also equicontinuous on $[0, T]$. Now the Arzelà-Ascoli theorem shows that $\mathscr{P}([0$, $1] \times \bar{\Omega})$ is compact in $C^{1}[0, T]$. Thus $\mathscr{P}$ is a compact operator.

Suppose that $x_{0}$ is a fixed point of the operator $\mathscr{P}(1, \cdot)$. Then

$$
x_{0}(t)=x_{0}(0)+\alpha\left(x_{0}\right)+\int_{0}^{t} \phi^{-1}\left(\phi\left(x_{0}^{\prime}(0)+\beta\left(x_{0}\right)\right)+\int_{0}^{s}\left(F x_{0}\right)(v) d v\right) d s
$$

Hence $\alpha\left(x_{0}\right)=0, \beta\left(x_{0}\right)=0$ and $x_{0}$ is a solution of (2.2). Therefore $x_{0}$ is a solution of the BVP (2.2), (1.4) and to prove our theorem, it suffices to show that

$$
D(\mathscr{I}-\mathscr{P}(1, \cdot), \Omega, 0) \neq 0
$$

where " $D$ " stands for the Leray-Schauder degree and $\mathscr{S}$ is the identity operator on $C^{1}[0, T]$. For this purpose let the compact operator $\mathscr{K}:[0,1] \times \bar{\Omega} \rightarrow C^{1}[0, T]$ be given by

$$
\mathscr{K}(\mu, x)(t)=x(0)+\alpha(x)-\mu \alpha(-x)+\left[x^{\prime}(0)+\beta(x)-\mu \beta(-x)\right] t
$$


6 General existence principles for nonlocal BVPs

Then $\mathscr{K}(1, \cdot)$ is odd (i.e., $\mathscr{K}(1,-x)=-\mathscr{K}(1, x)$ for $x \in \bar{\Omega})$ and

$$
\mathscr{K}(0, \cdot)=\mathscr{P}(0, \cdot)
$$

If $\mathscr{Y}\left(\mu_{0}, x_{0}\right)=x_{0}$ for some $\mu_{0} \in[0,1]$ and $x_{0} \in \bar{\Omega}$, then

$$
x_{0}(t)=x_{0}(0)+\alpha\left(x_{0}\right)-\mu_{0} \alpha\left(-x_{0}\right)+\left[x_{0}^{\prime}(0)+\beta\left(x_{0}\right)-\mu_{0} \beta\left(-x_{0}\right)\right] t, \quad t \in[0, T] .
$$

Therefore $x_{0}(t)=A_{0}+B_{0} t$ where $A_{0}=x_{0}(0)+\alpha\left(x_{0}\right)-\mu_{0} \alpha\left(-x_{0}\right), B_{0}=x_{0}^{\prime}(0)+\beta\left(x_{0}\right)-$ $\mu_{0} \beta\left(-x_{0}\right)$, and also $\alpha\left(x_{0}\right)-\mu_{0} \alpha\left(-x_{0}\right)=0, \beta\left(x_{0}\right)-\mu_{0} \beta\left(-x_{0}\right)=0$. Hence

$$
\begin{aligned}
& \alpha\left(A_{0}+B_{0} t\right)-\mu_{0} \alpha\left(-A_{0}-B_{0} t\right)=0, \\
& \beta\left(A_{0}+B_{0} t\right)-\mu_{0} \beta\left(-A_{0}-B_{0} t\right)=0 .
\end{aligned}
$$

Thus

$$
\left|A_{0}\right|<\Lambda_{0}, \quad\left|B_{0}\right|<\Lambda_{1}
$$

since $\left(A_{0}, B_{0}\right)$ is a solution of (2.6) with $\mu=\mu_{0}$. Then $\left\|x_{0}\right\|<\Lambda_{0}+\Lambda_{1} T$, $\left\|x_{0}^{\prime}\right\|<\Lambda_{1}$, which gives $x_{0} \notin \partial \Omega$. Now, by the Borsuk antipodal theorem and a homotopy property (see, e.g., $[6])$,

$$
D(\mathscr{I}-\mathscr{K}(0, \cdot), \Omega, 0)=D(\mathscr{I}-\mathscr{K}(1, \cdot), \Omega, 0) \neq 0 .
$$

Finally assume that $\mathscr{P}\left(\lambda_{*}, x_{*}\right)=x_{*}$ for some $\lambda_{*} \in[0,1]$ and $x_{*} \in \bar{\Omega}$. Then $x_{*}$ is a solution of the BVP (2.4) with $\lambda=\lambda_{*}$ and, by our assumptions, $\left\|x_{*}\right\|<S_{0}$ and $\left\|x_{*}^{\prime}\right\|<S_{1}$. Hence $x_{*} \notin \partial \Omega$ and a homotopy property yields

$$
D(\mathscr{I}-\mathscr{P}(0, \cdot), \Omega, 0)=D(\mathscr{I}-\mathscr{P}(1, \cdot), \Omega, 0)
$$

From the last equality, (2.15) and (2.19) it follows that (2.13) is true. Therefore the BVP (2.2), (1.4) has a solution.

Remark 2.2. Let the functionals $\alpha, \beta \in \mathscr{A}$ be linear. Then the system (2.6) has the form

$$
\begin{aligned}
& A \alpha(1)+B \alpha(t)=0 \\
& A \beta(1)+B \beta(t)=0
\end{aligned}
$$

and all of its solutions $(A, B)$ are bounded if and only if $\alpha(1) \beta(t)-\alpha(t) \beta(1) \neq 0$ (and then $(A, B)=(0,0))$.

Special cases of Theorem 2.1 are the general existence principles presented in [8] for the differential equation $\left(\phi\left(x^{\prime}\right)\right)^{\prime}=q(t) f\left(t, x, x^{\prime}\right)$ with the Dirichlet and mixed boundary conditions.

Now consider the singular functional-differential equation (1.3) where $\phi$ is an increasing homeomorphism from $\mathbb{R}$ onto $\mathbb{R}, f_{j} \in \operatorname{Car}\left([0, T] \times \mathscr{D}^{j}\right)$, the sets $\mathscr{D}^{j}=\mathscr{D}_{1}^{j} \times \mathscr{D}_{2}^{j} \subset \mathbb{R}^{2}$ 
are not necessarily closed, $f_{j}$ have singularities in their phase variables on the boundary $\partial \mathscr{D}^{j}$ of $\mathscr{D}^{j}(j=1,2,3)$ and $F_{i} \in \mathscr{H}^{\prime}(i=1,2)$. Also consider the sequence of regular differential equations (1.7) where $f_{j, n} \in \operatorname{Car}\left([0, T] \times \mathbb{R}^{2}\right), n \in \mathbb{N}, j=1,2,3$.

THeorem 2.3 (general existence principle for singular nonlocal BVP). Let $\alpha, \beta \in A$ A. Suppose there exist a bounded set $\Omega \subset C^{1}[0, T]$ such that

(i) for each $n \in \mathbb{N}$, the regular $B V P(1.7)$, (1.4) has a solution $x_{n} \in \Omega$,

(ii) the sequences $\left\{f_{j, n}\left(t, x_{n}(t), x_{n}^{\prime}(t)\right)\right\}$ are uniformly integrable on $[0, T]$ for $j=1,2,3$. Then we have

(a) there exist $x \in \bar{\Omega}$ and a subsequence $\left\{x_{k_{n}}\right\}$ of $\left\{x_{n}\right\}$ such that $\lim _{n \rightarrow \infty} x_{k_{n}}=x$ in $C^{1}([0$, $T]$ ),

(b) $x$ is a solution of the BVP (1.3), (1.4) if

$$
\lim _{n \rightarrow \infty} f_{j, k_{n}}\left(t, x_{k_{n}}(t), x_{k_{n}}^{\prime}(t)\right)=f_{j}\left(t, x(t), x^{\prime}(t)\right), \quad j=1,2,3,
$$

for almost all $t \in[0, T]$.

Proof. Since $\Omega$ is bounded in $C^{1}[0, T]$ and $\left\{x_{n}\right\} \subset \Omega$, there are positive constants $K$ and $r$ such that

$$
\left|\left(F_{1} x_{n}\right)(t)\right| \leq K, \quad\left|\left(F_{2} x_{n}\right)(t)\right| \leq K, \quad\left\|x_{n}\right\| \leq r, \quad\left\|x_{n}^{\prime}\right\| \leq r, \quad n \in \mathbb{N} .
$$

Now (ii) guarantees that for each $\varepsilon>0$ there exists $\delta>0$ such that for each $t_{1}, t_{2} \in[0, T]$, $\left|t_{2}-t_{1}\right|<\delta$, and $n \in \mathbb{N}$,

$$
\begin{aligned}
\left|\phi\left(x_{n}^{\prime}\left(t_{2}\right)\right)-\phi\left(x_{n}^{\prime}\left(t_{1}\right)\right)\right| \leq & \left|\int_{t_{1}}^{t_{2}}\right| f_{1, n}\left(t, x_{n}(t), x_{n}^{\prime}(t)\right)|d t| \\
& +K \sum_{j=2}^{3}\left|\int_{t_{1}}^{t_{2}}\right| f_{j, n}\left(t, x_{n}(t), x_{n}^{\prime}(t)\right)|d t|<\varepsilon .
\end{aligned}
$$

Therefore $\left\{\phi\left(x_{n}^{\prime}(t)\right)\right\}$ is equicontinuous on $[0, T]$, and then using (2.23) and the fact that $\phi$ is continuous and increasing on $\mathbb{R}$, we see that $\left\{x_{n}^{\prime}(t)\right\}$ is equicontinuous on $[0, T]$ as well. The Arzelà-Ascoli theorem guarantees the existence of a subsequence $\left\{x_{k_{n}}\right\}$ of $\left\{x_{n}\right\}$ converging in $C^{1}[0, T]$ to $x \in \bar{\Omega}$.

Suppose that $\lim _{n \rightarrow \infty} f_{j, k_{n}}\left(t, x_{k_{n}}(t), x_{k_{n}}^{\prime}(t)\right)=f_{j}\left(t, x(t), x^{\prime}(t)\right)$ for a.e. $t \in[0, T]$ and for $j=1,2,3$. By (ii), $\left\{f_{j, k_{n}}\left(t, x_{k_{n}}(t), x_{k_{n}}^{\prime}(t)\right)\right\}$ are uniformly integrable on $[0, T]$. Therefore, by Vitali's convergence theorem, $f_{j}\left(t, x(t), x^{\prime}(t)\right) \in L_{1}[0, T]$ for $j=1,2,3$ and also letting $n \rightarrow \infty$ in

$$
\begin{aligned}
\phi\left(x_{k_{n}}^{\prime}(t)\right)= & \phi\left(x_{k_{n}}^{\prime}(0)\right)+\int_{0}^{t} f_{1, k_{n}}\left(s, x_{k_{n}}(s), x_{k_{n}}^{\prime}(s)\right) d s \\
& +\int_{0}^{t} f_{2, k_{n}}\left(s, x_{k_{n}}(s), x_{k_{n}}^{\prime}(s)\right)\left(F_{1} x_{k_{n}}\right)(s) d s \\
& +\int_{0}^{t} f_{3, k_{n}}\left(s, x_{k_{n}}(s), x_{k_{n}}^{\prime}(s)\right)\left(F_{2} x_{k_{n}}\right)(s) d s
\end{aligned}
$$


8 General existence principles for nonlocal BVPs

for $t \in[0, T]$ and $n \in \mathbb{N}$, we have

$$
\begin{aligned}
\phi\left(x^{\prime}(t)\right)= & \phi\left(x^{\prime}(0)\right)+\int_{0}^{t} f_{1}\left(s, x(s), x^{\prime}(s)\right) d s \\
& +\int_{0}^{t} f_{2}\left(s, x(s), x^{\prime}(s)\right)\left(F_{1} x\right)(s) d s \\
& +\int_{0}^{t} f_{3}\left(s, x(s), x^{\prime}(s)\right)\left(F_{2} x\right)(s) d s, \quad t \in[0, T] .
\end{aligned}
$$

Consequently, $\phi\left(x^{\prime}\right) \in \mathrm{AC}[0, T]$ and $x$ is a solution of (1.3). In addition, since $\lim _{n \rightarrow \infty} x_{k_{n}}$ $=x$ in $C^{1}[0, T]$ and $\alpha$ and $\beta$ are continuous in $C^{1}[0, T]$, it follows that $\alpha(x)=0, \beta(x)=0$. Hence $x$ is a solution of the BVP (1.3), (1.4).

Remark 2.4. Let $f_{j}$ in (1.3) have singularities only at the value 0 of their phase variables and $f_{j, n}$ in (1.7) satisfy

$$
f_{j, n}(t, x, y)=f_{j}(t, x, y), \quad j=1,2,3
$$

for a.e. $t \in[0, T]$ and all $(x, y) \in \mathscr{D}^{j}, n \in \mathbb{N},|x| \geq 1 / n$ and $|y| \geq 1 / n$. Then the condition

$$
\lim _{n \rightarrow \infty} f_{j, k_{n}}\left(t, x_{k_{n}}(t), x_{k_{n}}^{\prime}(t)\right)=f_{j}\left(t, x(t), x^{\prime}(t)\right), \quad j=1,2,3
$$

for a.e. $t \in[0, T]$ is satisfied if $x$ and $x^{\prime}$ have a finite number of zeros.

Remark 2.5. The absolute continuity of the Lebesgue integral yields that condition (ii) in Theorem 2.3 is satisfied if there exists a function $\varphi \in L_{1}[0, T]$ such that

$$
\left|f_{j, n}\left(t, x_{n}(t), x_{n}^{\prime}(t)\right)\right| \leq \varphi(t)
$$

for a.e. $t \in[0, T]$ and each $n \in \mathbb{N}, j \in\{1,2,3\}$.

We now discuss the special case of (1.3) with $f_{1}=f$ and $f_{2}=f_{3}=0$, that is the differential equation

$$
\left(\phi\left(x^{\prime}(t)\right)\right)^{\prime}=f\left(t, x(t), x^{\prime}(t)\right)
$$

where $f \in \operatorname{Car}([0, T] \times(0, \infty) \times(\mathbb{R} \backslash\{0\}))$. Together with (2.30), we consider the sequence of regular differential equations

$$
\left(\phi\left(x^{\prime}(t)\right)\right)^{\prime}=f_{n}\left(t, x(t), x^{\prime}(t)\right)
$$

where $f_{n} \in \operatorname{Car}\left([0, T] \times \mathbb{R}^{2}\right)$ and

$$
f_{n}(t, x, y)=f(t, x, y) \quad \text { for a.e. } t \in[0, T] \text { and all } x \geq 1 / n,|y| \geq 1 / n \text {. }
$$

For the solvability of the singular BVP (2.30), (1.4) the following result holds. 
Theorem 2.6. Suppose that

(j) there exists $\gamma \in L_{1}[0, T]$ such that $f_{n}(t, x, y) \geq \gamma(t)>0$ for a.e. $t \in[0, T]$ and each $(x, y) \in \mathbb{R}^{2}, n \in \mathbb{N}$,

(ji) there exists a bounded set $\Omega \subset C^{1}[0, T]$ such that for each $n \in \mathbb{N}$, the $B V P$ (2.31), (1.4) has a solution $x_{n} \in \Omega, x_{n}\left(\xi_{n}\right)=x^{\prime}\left(\xi_{n}\right)=0$ where $\xi_{n} \in[0, T]$ and $\left\{x_{n}^{\prime}(t)\right\}$ is equicontinuous on $[0, T]$.

Then

(a) there exist $x \in \bar{\Omega}$ and a subsequence $\left\{x_{k_{n}}\right\}$ of $\left\{x_{n}\right\}$ such that $\lim _{n \rightarrow \infty} x_{k_{n}}=x$ in $C^{1}[0$, $T], x(\xi)=x^{\prime}(\xi)=0$ with $a \xi \in[0, T]$ and $x>0$ on $[0, T] \backslash\{\xi\}$.

(b) $x$ is a solution of the $B V P(2.30),(1.4)$ if to given $\varepsilon>0$, there exist $\delta_{\varepsilon} \in L_{1}[0, T]$ and $n_{\varepsilon} \in \mathbb{N}$ such that

$$
f_{k_{n}}\left(t, x_{k_{n}}(t), x_{k_{n}}^{\prime}(t)\right) \leq \delta_{\varepsilon}(t)
$$

for a.e. $t \in[0, T] \backslash[\xi-\varepsilon, \xi+\varepsilon]$ and each $n \geq n_{\varepsilon}$.

Proof. Since $\left(\phi\left(x_{n}^{\prime}(t)\right)\right)^{\prime}=f_{n}\left(t, x_{n}(t), x_{n}^{\prime}(t)\right) \geq \gamma(t)>0$ for a.e. $t \in[0, T]$ and each $n \in \mathbb{N}$, $x_{n}^{\prime}$ is increasing on $[0, T]$ and $x_{n}\left(\xi_{n}\right)=x_{n}^{\prime}\left(\xi_{n}\right)=0$ implies

$$
\begin{gathered}
x_{n}^{\prime}(t) \leq-\phi\left(\int_{t}^{\xi_{n}} \gamma(s) d s\right), \quad x_{n}(t) \geq \int_{t}^{\xi_{n}} \phi\left(\int_{s}^{\xi_{n}} \gamma(v) d v\right) d s, \quad t \in\left[0, \xi_{n}\right], \\
x_{n}^{\prime}(t) \geq \phi\left(\int_{\xi_{n}}^{t} \gamma(s) d s\right), \quad x_{n}(t) \geq \int_{\xi_{n}}^{t} \phi\left(\int_{\xi_{n}}^{s} \gamma(v) d v\right) d s, \quad t \in\left[\xi_{n}, T\right] .
\end{gathered}
$$

We know, by the assumptions, that $\left\{x_{n}\right\}$ is bounded in $C^{1}[0, T]$ and $\left\{x_{n}^{\prime}(t)\right\}$ is equicontinuous on $[0, T]$. Hence there exist a convergent subsequence $\left\{x_{k_{n}}\right\}$ converging to $x$ in $C^{1}[0, T]$ and a convergent subsequence $\left\{\xi_{k_{n}}\right\}$ converging to $\xi$ in $\mathbb{R}$. Then $x \in C^{1}[0, T]$, $\xi \in[0, T]$, and taking the limit as $n \rightarrow \infty$ in (2.34) with $k_{n}$ instead of $n$, we get

$$
\begin{gathered}
x^{\prime}(t) \leq-\phi\left(\int_{t}^{\xi} \gamma(s) d s\right), \quad x(t) \geq \int_{t}^{\xi} \phi\left(\int_{s}^{\xi} \gamma(v) d v\right) d s, \quad t \in[0, \xi], \\
x^{\prime}(t) \geq \phi\left(\int_{\xi}^{t} \gamma(s) d s\right), \quad x(t) \geq \int_{\xi}^{t} \phi\left(\int_{\xi}^{s} \gamma(v) d v\right) d s, \quad t \in[\xi, T] .
\end{gathered}
$$

Therefore $x(\xi)=x^{\prime}(\xi)=0, x>0,\left|x^{\prime}\right|>0$ on $[0, T] \backslash\{\xi\}$, and consequently (see (2.32))

$$
\lim _{n \rightarrow \infty} f_{k_{n}}\left(t, x_{k_{n}}(t), x_{k_{n}}^{\prime}(t)\right)=f\left(t, x(t), x^{\prime}(t)\right)
$$

for almost all $t \in[0, T]$.

From $\left\{\phi\left(x_{k_{n}}^{\prime}(0)\right)\right\}$ and $\left\{\phi\left(x_{k_{n}}^{\prime}(T)\right)\right\}$ being bounded,

$$
\phi\left(x_{k_{n}}^{\prime}(T)\right)-\phi\left(x_{k_{n}}^{\prime}(0)\right)=\int_{0}^{T} f_{k_{n}}\left(t, x_{k_{n}}(t), x_{k_{n}}^{\prime}(t)\right) d t, \quad n \in \mathbb{N}
$$


and $f_{k_{n}}\left(t, x_{k_{n}}(t), x_{k_{n}}^{\prime}(t)\right) \geq \gamma(t)>0$, it follows that $f\left(t, x(t), x^{\prime}(t)\right) \in L_{1}[0, T]$, by Fatou's theorem. If the condition in (b) is satisfied, then letting $n \rightarrow \infty$ in

$$
\begin{aligned}
& \phi\left(x_{k_{n}}^{\prime}(t)\right)=\phi\left(x_{k_{n}}^{\prime}(0)\right)+\int_{0}^{t} f_{k_{n}}\left(s, x_{k_{n}}(s), x_{k_{n}}^{\prime}(s)\right) d s, \\
& \phi\left(x_{k_{n}}^{\prime}(t)\right)=\phi\left(x_{k_{n}}^{\prime}(T)\right)-\int_{T}^{t} f_{k_{n}}\left(s, x_{k_{n}}(s), x_{k_{n}}^{\prime}(s)\right) d s,
\end{aligned}
$$

we obtain

$$
\begin{array}{ll}
\phi\left(x_{n}^{\prime}(t)\right)=\phi\left(x^{\prime}(0)\right)+\int_{0}^{t} f\left(s, x(s), x^{\prime}(s)\right) d s, & t \in[0, \xi), \\
\phi\left(x^{\prime}(t)\right)=\phi\left(x^{\prime}(T)\right)-\int_{T}^{t} f\left(s, x(s), x^{\prime}(s)\right) d s, & t \in(\xi, T]
\end{array}
$$

by the Lebesgue dominated convergence theorem. Now using $f\left(t, x(t), x^{\prime}(t)\right) \in L_{1}[0, T]$, we have $\phi\left(x^{\prime}\right) \in \mathrm{AC}[0, T]$ and $x$ is a solution of (2.30). Also from $\alpha\left(x_{k_{n}}\right)=0, \beta\left(x_{k_{n}}\right)=0$ and the continuity of $\alpha$ and $\beta$ on $C^{1}[0, T]$ it follows $\alpha(x)=0, \beta(x)=0$. Hence $x$ is a solution of the BVP (2.30), (1.4).

\section{Application to singular nonlocal BVPs with positive nonlinearities}

3.1. Introduction. Denote by $\mathscr{B}$ the set of all functionals $\alpha: C^{1}[0, T] \rightarrow \mathbb{R}$ which are

(i) continuous,

(ii) sign preserving with respect to the derivative of functions in the following sense: $x \in C^{1}[0, T], \varepsilon x^{\prime}>0$ on $[0, T]$ where $\varepsilon \in\{-1,1\} \Rightarrow \varepsilon \alpha(x)>0$,

(iii) bounded that is $\Omega \subset C^{1}[0, T]$ bounded $\Rightarrow \alpha(\Omega)$ bounded.

Of course, $\mathscr{B} \subset \mathscr{A}$ where $\mathscr{A}$ is defined in Section 1.

Remark 3.1. If $\alpha \in \mathscr{B}$ then $\alpha(A)=0$ for each $A \in \mathbb{R}$. Indeed, let $\alpha \in \mathscr{B}, A \in \mathbb{R}$ and set $x_{n}(t)=A+t / n, y_{n}(t)=A-t / n$ for $t \in[0, T]$ and $n \in \mathbb{N}$. Then $\alpha\left(y_{n}\right)<0<\alpha\left(x_{n}\right)$ by (ii), and $\left\{x_{n}\right\},\left\{y_{n}\right\}$ are convergent in $C^{1}[0, T]$ to $A$. Thus $0 \geq \lim _{n \rightarrow \infty} \alpha\left(y_{n}\right)=\alpha(A)$ and $0 \leq$ $\lim _{n \rightarrow \infty} \alpha\left(x_{n}\right)=\alpha(A)$ yield $\alpha(A)=0$.

Example 3.2. Let $\gamma: C^{1}[0, T] \rightarrow[0, T]$ be a continuous functional, $q, r \in C^{0}(\mathbb{R}), q$ be positive, $u r(u)>0$ for $u \neq 0$ and $0 \leq t_{1}<t_{2}<\cdots<t_{n} \leq T$. Then the functionals

$$
\alpha_{1}(x)=x^{\prime}(\gamma(x)), \quad \alpha_{2}(x)=\int_{0}^{T} q(x(t)) r\left(x^{\prime}(t)\right) d t, \quad \alpha_{3}(x)=\sum_{j=1}^{n} q\left(x\left(t_{j}\right)\right) r\left(x^{\prime}\left(t_{j}\right)\right)
$$

and all their linear combination with positive coefficients belong to the set $\mathscr{B}$.

We discuss the singular nonlocal boundary value problems

$$
\begin{gathered}
\left(\phi\left(x^{\prime}(t)\right)\right)^{\prime}=f\left(t, x(t), x^{\prime}(t)\right), \\
\min \{x(t): 0 \leq t \leq T\}=0, \quad \alpha(x)=0,
\end{gathered}
$$

where $\phi \in C^{0}(\mathbb{R}), \alpha \in \mathscr{B}, f \in \operatorname{Car}([0, T] \times(0, \infty) \times(\mathbb{R} \backslash\{0\}))$ is positive and $f$ may be 
singular at the value 0 of both its phase variables in the following sense $\lim _{x \rightarrow 0^{+}} f(t, x, y)=$ $\infty$ for a.e. $t \in[0, T]$ and each $y \in \mathbb{R} \backslash\{0\}, \lim _{y \rightarrow 0} f(t, x, y)=\infty$ for a.e. $t \in[0, T]$ and each $x \in(0, \infty)$. Equation (3.2) is the special case of (1.3) with $F_{1}=F_{2}=0$.

A function $x \in C^{1}[0, T]$ is said to be a solution of the BVP (3.2), (3.3) if $\phi\left(x^{\prime}\right) \in$ $\mathrm{AC}[0, T], x$ satisfies (3.3) and for a.e. $t \in[0, T]$ fulfills (3.2).

We give conditions on the functions $\phi$ and $f$ in (3.2) which guarantee the solvability of the BVP (3.2), (3.3) for each $\alpha \in \mathscr{B}$ in (3.3). Existence results are based on regularization and sequential techniques and use our general existence principles (Theorems 2.1 and 2.6). Notice that any solution of the BVP (3.2), (3.3) and its derivative "go through" singularities of $f$ somewhere inside of $[0, T]$.

Throughout this section we will use the following assumptions.

$\left(\mathrm{H}_{1}\right) \phi \in C^{0}(\mathbb{R})$ is odd and increasing on $\mathbb{R}, \lim _{x \rightarrow \infty} \phi(x)=\infty$;

$\left(\mathrm{H}_{2}\right) f \in \operatorname{Car}([0, T] \times(0, \infty) \times(\mathbb{R} \backslash\{0\}))$ and there exists $\delta \in L_{1}[0, T]$, such that for a.e. $t \in[0, T]$ and each $(x, y) \in(0, \infty) \times(\mathbb{R} \backslash\{0\})$,

$$
0<\delta(t) \leq f(t, x, y)
$$

$\left(\mathrm{H}_{3}\right)$ For a.e. $t \in[0, T]$ and each $(x, y) \in(0, \infty) \times(\mathbb{R} \backslash\{0\})$,

$$
f(t, x, y) \leq\left(h_{1}(x)+h_{2}(x)\right)\left[\omega_{1}(|\phi(y)|)+\omega_{2}(|\phi(y)|)\right],
$$

with $h_{1}, \omega_{1} \in C^{0}[0, \infty)$ positive and nondecreasing, $h_{2}, \omega_{2} \in C^{0}(0, \infty)$ positive and nonincreasing, $h_{1}+h_{2}$ and $\omega_{1}+\omega_{2}$ nonincreasing on a neighbourhood of 0 ,

$$
\begin{gathered}
\int_{0}^{1} h_{2}(s) d s<\infty, \\
\liminf _{x \rightarrow \infty} \frac{G(x)}{H(T x)}>1,
\end{gathered}
$$

where

$$
G(x)=\int_{0}^{\phi(x)} \frac{\phi^{-1}(s)}{\omega_{1}(s)+\omega_{2}(s)} d s, \quad H(x)=\int_{0}^{x}\left(h_{1}(s)+h_{2}(s)\right) d s
$$

for $x \in[0, \infty)$.

3.2. Auxiliary regular BVPs. Let assumption $\left(\mathrm{H}_{2}\right)$ be satisfied. For each $n \in \mathbb{N}$, define the function $f_{n} \in \operatorname{Car}\left([0, T] \times \mathbb{R}^{2}\right)$ by the formula

$$
f_{n}(t, x, y)= \begin{cases}f(t, x, y) & \text { for } t \in[0, T], x \geq \frac{1}{n},|y| \geq \frac{1}{n} \\ f\left(t, \frac{1}{n}, y\right) & \text { for } t \in[0, T], x<\frac{1}{n},|y| \geq \frac{1}{n} \\ \frac{n}{2}\left[f_{n}\left(t, x, \frac{1}{n}\right)\left(y+\frac{1}{n}\right)\right. & \text { for } t \in[0, T], x \in \mathbb{R},|y|<\frac{1}{n} . \\ \left.-f_{n}\left(t, x,-\frac{1}{n}\right)\left(y-\frac{1}{n}\right)\right]\end{cases}
$$


Then $\left(\mathrm{H}_{2}\right)$ gives

$$
0<\delta(t) \leq f_{n}(t, x, y), \quad(t, x, y) \in[0, T] \times \mathbb{R}^{2}, n \in \mathbb{N} .
$$

Since $h_{1}+h_{2}$ and $\omega_{1}+\omega_{2}$ are nonincreasing on a neighbourhood of 0 by $\left(\mathrm{H}_{3}\right)$, there exists $n_{*} \in \mathbb{N}$ such that $h_{1}(1 / n)+h_{2}(1 / n) \leq h_{1}(x)+h_{2}(x)$ and $\omega_{1}(\phi(1 / n))+\omega_{2}(\phi(1 / n)) \leq$ $\omega_{1}(\phi(x))+\omega_{2}(\phi(x))$ for $n \geq n_{*}$ and $x \in(0,1 / n]$. Hence $\left(\mathrm{H}_{3}\right)$ yields

$$
\lambda f_{n}(t, x, y) \leq\left(h_{1}(|x|)+h_{2}(|x|)\right)\left[\omega_{1}(|\phi(y)|)+\omega_{2}(|\phi(y)|)\right]
$$

for a.e. $t \in[0, T]$ and each $(x, y) \in \mathbb{R}_{0}^{2}, \lambda \in[0,1], n \in \mathbb{N}_{*}$, where $\mathbb{N}_{*}=\{n: n \in \mathbb{N}, n \geq$ $\left.n_{*}\right\}$.

Consider the family of regular differential equations

$$
\left(\phi\left(x^{\prime}(t)\right)\right)^{\prime}=\lambda f_{n}\left(t, x(t), x^{\prime}(t)\right)
$$

depending on the parameters $\lambda \in[0,1]$ and $n \in \mathbb{N}_{*}$.

We need some preliminary results given in the next two lemmas.

Lemma 3.3. Let $\alpha \in \mathscr{B}$ and $\alpha(x)-\lambda \alpha(-x)=0$ for some $x \in C^{1}[0, T]$ and $\lambda \in[0,1]$. Then $x^{\prime}(\xi)=0$ for $a \xi \in[0, T]$.

Proof. If not, then $\varepsilon x^{\prime}>0$ on $[0, T]$ where $\varepsilon \in\{-1,1\}$. Therefore $\varepsilon \alpha(x)>0$ and $\varepsilon \alpha(-x)<$ 0 . Hence $\varepsilon[\alpha(x)-\lambda \alpha(-x)]>0$, which is impossible.

Lemma 3.4. Let $\alpha \in \mathscr{B}$. Then for each $\mu \in[0,1]$, the system

$$
\begin{gathered}
\alpha(A+B t)-\mu \alpha(-A-B t)=0, \\
\min \{A+B t: 0 \leq t \leq T\}-\mu \min \{-A-B t: 0 \leq t \leq T\}=0,
\end{gathered}
$$

has the unique solution $(A, B)=(0,0)$ in $\mathbb{R}^{2}$.

Proof. Fix $\mu \in[0,1]$. Let $\left(A_{0}, B_{0}\right) \in \mathbb{R}^{2}$ be a solution of (3.13). If $B_{0} \neq 0$, then from the properties of $\alpha$ it follows that $\nu \alpha\left(A_{0}+B_{0} t\right)>0$ and $\nu \alpha\left(-A_{0}-B_{0} t\right)<0$ where $\nu=\operatorname{sign} B_{0}$. Therefore $\nu\left[\alpha\left(A_{0}+B_{0} t\right)-\mu \alpha\left(-A_{0}-B_{0} t\right)\right]>0$, contrary to $\left[\alpha\left(A_{0}+B_{0} t\right)-\mu \alpha\left(-A_{0}-B_{0} t\right)\right]$ $=0$. Hence $B_{0}=0$. Then

$$
0=\min \left\{A_{0}+B_{0} t: 0 \leq t \leq T\right\}-\mu \min \left\{-A_{0}-B_{0} t: 0 \leq t \leq T\right\}=(1+\mu) A_{0},
$$

and $A_{0}=0$. We have proved that $(0,0)$ is the unique solution of $(3.13)$ for each $\mu \in[0,1]$.

Now we give a priori bounds for solutions of the BVP $(3.12)_{n}^{\lambda},(3.3)$.

Lemma 3.5. Let assumptions $\left(H_{1}\right)$ and $\left(H_{2}\right)$ be satisfied. Then

$$
\begin{gathered}
\left|x^{\prime}(t)\right| \geq \phi^{-1}\left(\lambda \int_{t}^{\xi_{x}} \delta(s) d s\right), \quad x(t) \geq \int_{t}^{\xi_{x}} \phi^{-1}\left(\lambda \int_{s}^{\xi_{x}} \delta(v) d v\right) d s, \quad t \in\left[0, \xi_{x}\right], \\
x^{\prime}(t) \geq \phi^{-1}\left(\lambda \int_{\xi_{x}}^{t} \delta(s) d s\right), \quad x(t) \geq \int_{\xi_{x}}^{t} \phi^{-1}\left(\lambda \int_{\xi_{x}}^{s} \delta(v) d v\right) d s, \quad t \in\left[\xi_{x}, T\right],
\end{gathered}
$$


for any solution $x$ of the $B V P(3.12)_{n}^{\lambda}$, (3.3) with $\lambda \in(0,1]$ and $n \in \mathbb{N}_{*}$, where $\xi_{x}$ is the unique zero of $x$ (and also $x^{\prime}$ ) in $[0, T]$. If $\lambda=0$, then $x=0$ is the unique solution of the $B V P$ $(3.12)_{n}^{0},(3.3)$.

Proof. Let $x$ be a solution of the BVP $(3.12)_{n}^{\lambda}$, (3.3) with some $\lambda \in(0,1]$ and $n \in \mathbb{N}_{*}$. Since

$$
\left(\phi\left(x^{\prime}(t)\right)\right)^{\prime} \geq \lambda \delta(t)>0 \quad \text { for a.e. } t \in[0, T]
$$

by (3.10), $\phi\left(x^{\prime}\right)$ is increasing on $[0, T]$ and so $x^{\prime}$ is as well. Now $\alpha(x)=0$ and Lemma 3.3 imply that $x^{\prime}\left(\xi_{x}\right)=0$ for a unique $\xi_{x} \in[0, T]$. Therefore $x^{\prime}<0$ on $\left[0, \xi_{x}\right)$ (if $\xi_{x}>0$ ) and $x^{\prime}>0$ on $\left(\xi_{x}, T\right]$ (if $\left.\xi_{x}<T\right)$. From the condition $\min \{x(t): 0 \leq t \leq T\}=0$ it follows that $x\left(\xi_{x}\right)=0$ and $x>0$ on $[0, T] \backslash\left\{\xi_{x}\right\}$. Integrating (3.17) from $t \in\left[0, \xi_{x}\right.$ ) to $\xi_{x}$ (if $\xi_{x}>0$ ) and using the fact that $\phi^{-1}$ is an odd function on $\mathbb{R}$, we get

$$
\left|x^{\prime}(t)\right| \geq \phi^{-1}\left(\lambda \int_{t}^{\xi_{x}} \delta(s) d s\right)
$$

Hence

$$
x(t)=\int_{t}^{\xi_{x}}\left|x^{\prime}(s)\right| d s \geq \int_{t}^{\xi_{x}} \phi^{-1}\left(\lambda \int_{s}^{\xi_{x}} \delta(v) d v\right) d s
$$

for $t \in\left[0, \xi_{x}\right]$. Integrating (3.17) now from $\xi_{x}$ to $t \in\left(\xi_{x}, T\right]$ (if $\xi_{x}<T$ ) yields

$$
x^{\prime}(t) \geq \phi^{-1}\left(\lambda \int_{\xi_{x}}^{t} \delta(s) d s\right)
$$

and therefore

$$
x(t)=\int_{\xi_{x}}^{t} x^{\prime}(s) d s \geq \int_{\xi_{x}}^{t} \phi^{-1}\left(\lambda \int_{\xi_{x}}^{s} \delta(v) d v\right) d s
$$

for $t \in\left[\xi_{x}, T\right]$. The validity of (3.15) follows from (3.18) and (3.19) and that of (3.16) from (3.20) and (3.21).

It is clear that $x=0$ is the unique solution of the BVP $(3.12)_{n}^{0},(3.3)$.

Remark 3.6. If $\left(\mathrm{H}_{1}\right)$ and $\left(\mathrm{H}_{2}\right)$ are satisfied, then (3.15) and (3.16) show that $x>0$ on $[0, T] \backslash\left\{\xi_{x}\right\}$ for any solution $x$ of the $\operatorname{BVP}(3.12)_{n}^{\lambda},(3.3)$ with $\lambda \in(0,1]$ and $n \in \mathbb{N}_{*}$, where $\xi_{x} \in[0, T]$ is the unique zero of $x$.

Lemma 3.7. Let assumptions $\left(H_{1}\right)-\left(H_{3}\right)$ be satisfied. Then there are positive constants $M_{0}$ and $M_{1}$ independent of $\lambda$ and $n$ such that

$$
\|x\|<M_{0}, \quad\left\|x^{\prime}\right\|<M_{1}
$$

for any solution $x$ to the $B V P(3.12)_{n}^{\lambda}$, (3.3) with $\lambda \in[0,1]$ and $n \in \mathbb{N}_{*}$. 
Proof. Fix $\lambda \in(0,1]$ and $n \in \mathbb{N}_{*}$. Let $x$ be a solution of the BVP $(3.12)_{n}^{\lambda},(3.3)$. We know that (see Remark 3.6 and the proof of Lemma 3.5) $x\left(\xi_{x}\right)=x^{\prime}\left(\xi_{x}\right)=0$ for a unique $\xi_{x} \in$ $[0, T], x^{\prime}<0$ on $\left[0, \xi_{x}\right)\left(\right.$ if $\left.\xi_{x}>0\right), x^{\prime}>0$ on $\left(\xi_{x}, T\right]\left(\right.$ if $\left.\xi_{x}<T\right)$ and $x^{\prime}$ is increasing on $[0, T]$. Hence

$$
\left\|x^{\prime}\right\|=\max \left\{\left|x^{\prime}(0)\right|, x^{\prime}(T)\right\}
$$

Also, by (3.11),

$$
\left(\phi\left(x^{\prime}(t)\right)\right)^{\prime} \leq\left[h_{1}(x(t))+h_{2}(x(t))\right]\left[\omega_{1}\left(\left|\phi\left(x^{\prime}(t)\right)\right|\right)+\omega_{2}\left(\left|\phi\left(x^{\prime}(t)\right)\right|\right)\right]
$$

for a.e. $t \in[0, T]$. Integrating from $t \in\left[0, \xi_{x}\right)$ to $\xi_{x}$ the inequality

$$
\frac{\left(\phi\left(x^{\prime}(t)\right)\right)^{\prime} x^{\prime}(t)}{\omega_{1}\left(-\phi\left(x^{\prime}(t)\right)\right)+\omega_{2}\left(-\phi\left(x^{\prime}(t)\right)\right)} \geq\left[h_{1}(x(t))+h_{2}(x(t))\right] x^{\prime}(t)
$$

we get

$$
G\left(\left|x^{\prime}(t)\right|\right) \leq H(x(t)), \quad t \in\left[0, \xi_{x}\right],
$$

where $G$ and $H$ are given in (3.8). Integrating the inequality (see (3.24))

$$
\frac{\left(\phi\left(x^{\prime}(t)\right)\right)^{\prime} x^{\prime}(t)}{\omega_{1}\left(\phi\left(x^{\prime}(t)\right)\right)+\omega_{2}\left(\phi\left(x^{\prime}(t)\right)\right)} \leq\left[h_{1}(x(t))+h_{2}(x(t))\right] x^{\prime}(t)
$$

over $\left[\xi_{x}, t\right] \subset\left[\xi_{x}, T\right]$, we get

$$
G\left(x^{\prime}(t)\right) \leq H(x(t)), \quad t \in\left[\xi_{x}, T\right] .
$$

Since $\|x\| \leq T\left\|x^{\prime}\right\|$, from (3.23), (3.26) with $t=0$ and (3.28) with $t=T$, we obtain

$$
G\left(\left\|x^{\prime}\right\|\right)=G\left(\max \left\{\left|x^{\prime}(0)\right|, x^{\prime}(T)\right\}\right) \leq H\left(T\left\|x^{\prime}\right\|\right) .
$$

By (3.7), there exists a positive constant $M_{1}$ such that $G(u) / H(T u)>1$ holds for all $u \geq$ $M_{1}$. Therefore (see (3.29)) $\left\|x^{\prime}\right\|<M_{1}$ and then $\|x\|<M_{0}$ where $M_{0}=T M_{1}$.

If $\lambda=0$, then $x=0$ is the unique solution of the BVP $(3.12)_{n}^{0},(3.3)$. This solution also satisfies (3.22).

Finally, we state and prove an existence result for the BVP $(3.12)_{n}^{1},(3.3)$.

Lemma 3.8. Suppose that assumptions $\left(H_{1}\right)-\left(H_{3}\right)$ are satisfied. Then the BVP $(3.12)_{n}^{1},(3.3)$ has a solution $x_{n}$ for each $n \in \mathbb{N}_{*}$ and

$$
\left\|x_{n}\right\|<M_{0}, \quad\left\|x_{n}^{\prime}\right\|<M_{1}, \quad n \in \mathbb{N}_{*},
$$

where $M_{0}$ and $M_{1}$ are positive constants.

Proof. By Lemma 3.7, there exists positive constants $M_{0}$ and $M_{1}$ such that any solution $x$ of the BVP $(3.12)_{n}^{\lambda},(3.3)$ with $n \in \mathbb{N}_{*}$ and $\lambda \in[0,1]$ satisfies (3.22). Fix $n \in \mathbb{N}_{*}$. Now using Lemma 3.4, the solvability of the BVP (3.12) ${ }_{n}^{1}$, (3.3) follows from Theorem 2.1 where we set $(F x)(t)=f_{n}\left(t, x(t), x^{\prime}(t)\right)$ for $x \in C^{1}[0, T]$ in (2.2). 
3.3. Existence result and an example. In the proof of the solvability of the BVP (3.2), (3.3), we will need the following result.

Lemma 3.9. Let assumptions $\left(H_{1}\right)-\left(H_{3}\right)$ be satisfied and let $x_{n}$ be a solution of the BVP $(3.12)_{n}^{1}$, (3.3) for $n \in \mathbb{N}_{*}$. Then the sequence $\left\{x_{n}^{\prime}\right\}_{n \in \mathbb{N}_{*}}$ is equicontinuous on $[0, T]$.

Proof. By Lemma 3.8 and (3.3),

$$
0 \leq x_{n}(t)<M_{0}, \quad\left\|x_{n}^{\prime}\right\|<M_{1}, \quad t \in[0, T], n \in \mathbb{N}_{*}
$$

where $M_{0}$ and $M_{1}$ are positive constants. Also $x_{n}\left(\xi_{n}\right)=x_{n}^{\prime}\left(\xi_{n}\right)=0$ for a unique $\xi_{n} \in[0, T]$, $x_{n}^{\prime}<0$ on $\left[0, \xi_{n}\right)$ (if $\xi_{n}>0$ ), $x_{n}^{\prime}>0$ on $\left(\xi_{n}, T\right]$ (if $\xi_{n}<T$ ) and $x_{n}^{\prime}$ is increasing on $[0, T]$. Integrating (see (3.25) and (3.27))

$$
\frac{\left(\phi\left(x_{n}^{\prime}(t)\right)\right)^{\prime} x_{n}^{\prime}(t)}{\omega_{1}\left(-\phi\left(x_{n}^{\prime}(t)\right)\right)+\omega_{2}\left(-\phi\left(x_{n}^{\prime}(t)\right)\right)} \geq\left[h_{1}\left(x_{n}(t)\right)+h_{2}\left(x_{n}(t)\right)\right] x_{n}^{\prime}(t)
$$

from $\eta_{1}$ to $\eta_{2}, 0 \leq \eta_{1} \leq \eta_{2} \leq \xi_{n}$, and

$$
\frac{\left(\phi\left(x_{n}^{\prime}(t)\right)\right)^{\prime} x_{n}^{\prime}(t)}{\omega_{1}\left(\phi\left(x_{n}^{\prime}(t)\right)\right)+\omega_{2}\left(\phi\left(x_{n}^{\prime}(t)\right)\right)} \leq\left[h_{1}\left(x_{n}(t)\right)+h_{2}\left(x_{n}(t)\right)\right] x_{n}^{\prime}(t)
$$

from $\eta_{3}$ to $\eta_{4}, \xi_{n} \leq \eta_{3} \leq \eta_{4} \leq T$, we get

$$
\begin{gathered}
(0 \leq) G\left(-x_{n}^{\prime}\left(\eta_{1}\right)\right)-G\left(-x_{n}^{\prime}\left(\eta_{2}\right)\right) \leq H\left(x_{n}\left(\eta_{1}\right)\right)-H\left(x_{n}\left(\eta_{2}\right)\right), \\
(0 \leq) G\left(x_{n}^{\prime}\left(\eta_{4}\right)\right)-G\left(x_{n}^{\prime}\left(\eta_{3}\right)\right) \leq H\left(x_{n}\left(\eta_{4}\right)\right)-H\left(x_{n}\left(\eta_{3}\right)\right),
\end{gathered}
$$

respectively. Since $\left\{x_{n}\right\}_{n \in \mathbb{N}_{*}}$ is bounded in $C^{0}[0, T]$ and equicontinouts on $[0, T]$ by $(3.31)$ and $H$ is continuous and increasing on $[0, T],\left\{H\left(x_{n}(t)\right)\right\}_{n \in \mathbb{N}_{*}}$ is equicontinuous on $[0, T]$. Hence to given $\varepsilon>0$ there exists $\gamma>0$ such that for each $t_{1}, t_{2} \in[0, T],\left|t_{1}-t_{2}\right|<\gamma$, we have

$$
\left|H\left(x_{n}\left(t_{2}\right)\right)-H\left(x_{n}\left(t_{1}\right)\right)\right|<\varepsilon, \quad n \in \mathbb{N}_{*} .
$$

We now show that $\left\{\hat{G}\left(x_{n}^{\prime}(t)\right)\right\}_{n \in \mathbb{N}_{*}}$ is equicontinuous on $[0, T]$, where $\hat{G} \in C^{0}(\mathbb{R})$ is defined by the formula

$$
\hat{G}(u)= \begin{cases}G(u) & \text { for } u \in[0, \infty) \\ -G(-u) & \text { for } u \in(-\infty, 0) .\end{cases}
$$

Let $0 \leq t_{1} \leq t_{2} \leq T, t_{2}-t_{1}<\gamma$. If $t_{2} \leq \xi_{n}$, then (see (3.34) with $\left.\eta_{1}=t_{1}, \eta_{2}=t_{2}\right)$

$$
(0 \leq) \hat{G}\left(x_{n}^{\prime}\left(t_{2}\right)\right)-\hat{G}\left(x_{n}^{\prime}\left(t_{1}\right)\right) \leq H\left(x_{n}\left(t_{1}\right)\right)-H\left(x_{n}\left(t_{2}\right)\right)<\varepsilon,
$$

and if $\xi_{n} \leq t_{1}$, then (see (3.35) with $\left.\eta_{3}=t_{1}, \eta_{4}=t_{2}\right)$

$$
(0 \leq) \hat{G}\left(x_{n}^{\prime}\left(t_{2}\right)\right)-\hat{G}\left(x_{n}^{\prime}\left(t_{1}\right)\right) \leq H\left(x_{n}\left(t_{2}\right)\right)-H\left(x_{n}\left(t_{1}\right)\right)<\varepsilon .
$$


Finally, let $t_{1}<\xi_{n}<t_{2}$. Then (see (3.34) with $\eta_{1}=t_{1}, \eta_{2}=\xi_{n}$ and (see (3.35) with $\eta_{3}=\xi_{n}$, $\left.\eta_{4}=t_{2}\right)$

$$
(0 \leq)-\hat{G}\left(x_{n}^{\prime}\left(t_{1}\right)\right) \leq H\left(x_{n}\left(t_{1}\right)\right)<\varepsilon, \quad(0 \leq) \hat{G}\left(x_{n}^{\prime}\left(t_{2}\right)\right) \leq H\left(x_{n}\left(t_{2}\right)\right)<\varepsilon .
$$

From (3.38)-(3.40) it follows

$$
0 \leq \hat{G}\left(x_{n}^{\prime}\left(t_{2}\right)\right)-\hat{G}\left(x_{n}^{\prime}\left(t_{1}\right)\right)<2 \varepsilon,
$$

and consequently $\left\{\hat{G}\left(x_{n}^{\prime}(t)\right)\right\}_{n \in \mathbb{N}_{*}}$ is equicontinuous on $[0, T]$. Since $\left\{x_{n}^{\prime}\right\}_{n \in \mathbb{N}_{*}}$ is bounded in $C^{0}[0, T]$ and $\hat{G}$ is continuous and increasing on $\mathbb{R},\left\{x_{n}^{\prime}(t)\right\}_{n \in \mathbb{N}_{*}}$ is equicontinuous on $[0, T]$.

Theorem 3.10. Suppose assumptions $\left(H_{1}\right)-\left(H_{3}\right)$ are satisfied. Then the BVP (3.2), (3.3) has a solution for each $\alpha \in \mathscr{B}$ in (3.3).

Proof. Fix $\alpha \in \mathscr{B}$. By Lemma 3.8, there exists a solution $x_{n}$ of the BVP $(3.12)_{n}^{1},(3.3)$ for any $n \in \mathbb{N}_{*}$ and the inequalities (3.31) are true where $M_{0}$ and $M_{1}$ are positive constants. Also Lemma 3.5 shows that

$$
\begin{gathered}
\left|x_{n}^{\prime}(t)\right| \geq \phi^{-1}\left(\int_{t}^{\xi_{n}} \delta(s) d s\right), \quad x_{n}(t) \geq \int_{t}^{\xi_{n}} \phi^{-1}\left(\int_{s}^{\xi_{n}} \delta(v) d v\right) d s, \quad t \in\left[0, \xi_{n}\right], \\
x_{n}^{\prime}(t) \geq \phi^{-1}\left(\int_{\xi_{n}}^{t} \delta(s) d s\right), \quad x_{n}(t) \geq \int_{\xi_{n}}^{t} \phi^{-1}\left(\int_{\xi_{n}}^{s} \delta(v) d v\right) d s, \quad t \in\left[\xi_{n}, T\right],
\end{gathered}
$$

where $\xi_{n} \in[0, T]$ is the unique zero of $x_{n}$. In addition the sequence $\left\{x_{n}^{\prime}(t)\right\}_{n \in \mathbb{N}_{*}}$ is equicontinuous on $[0, T]$ by Lemma 3.9. Hence Theorem 2.6 (see assertion (a)) guarantees the existence of a subsequence $\left\{x_{k_{n}}\right\}$ of $\left\{x_{n}\right\}$ converging in $C^{1}[0, T]$ to $x$ and $x(\xi)=x^{\prime}(\xi)=0$ for a unique $\xi \in[0, T]$. Letting $n \rightarrow \infty$ in (3.42) with $x_{k_{n}}$ instead of $x_{n}$ and $\xi_{k_{n}}$ instead of $\xi_{n}$, we get

$$
\begin{gathered}
\left|x^{\prime}(t)\right| \geq \phi^{-1}\left(\int_{t}^{\xi} \delta(s) d s\right), \quad x(t) \geq \int_{t}^{\xi} \phi^{-1}\left(\int_{s}^{\xi} \delta(v) d v\right) d s, \quad t \in[0, \xi], \\
x^{\prime}(t) \geq \phi^{-1}\left(\int_{\xi}^{t} \delta(s) d s\right), \quad x(t) \geq \int_{\xi}^{t} \phi^{-1}\left(\int_{\xi}^{s} \delta(v) d v\right) d s, \quad t \in[\xi, T] .
\end{gathered}
$$

Fix $\varepsilon>0$. Since $\lim _{n \rightarrow \infty} x_{k_{n}}=x$ in $C^{1}[0, T]$ and $x>0,\left|x^{\prime}\right|>0$ on $[0, T] \backslash\{\xi\}$ by (3.43), there exists $n_{\varepsilon} \in \mathbb{N}_{*}$ such that

$$
x_{k_{n}}(t) \geq \mu_{\varepsilon}^{0}, \quad\left|x_{k_{n}}^{\prime}(t)\right| \geq \mu_{\varepsilon}^{1}, \quad t \in[0, T] \backslash[\xi-\varepsilon, \xi+\varepsilon], n \geq n_{\varepsilon},
$$

where $\mu_{\varepsilon}^{0}=(1 / 2) \min \{x(t): t \in[0, T] \backslash[\xi-\varepsilon, \xi+\varepsilon]\}$ and $\mu_{\varepsilon}^{1}=(1 / 2) \min \left\{\left|x^{\prime}(t)\right|: t \in[0\right.$, $T] \backslash[\xi-\varepsilon, \xi+\varepsilon]\}$. Then

$$
(0<) f_{k_{n}}\left(t, x_{k_{n}}(t), x_{k_{n}}^{\prime}(t)\right) \leq \delta_{\varepsilon} \quad \text { for a.e. } t \in[0, T]
$$

where $\delta_{\varepsilon}=\left(h_{1}\left(M_{0}\right)+h_{2}\left(\mu_{\varepsilon}^{0}\right)\right)\left[\omega\left(\phi\left(M_{1}\right)\right)+\omega_{2}\left(\phi\left(\mu_{\varepsilon}^{1}\right)\right)\right]$. Hence $x$ is a solution of the BVP (3.2), (3.3) by Theorem 2.6 (see assertion (b)). 
Example 3.11. Consider the differential equation

$$
\left(\phi_{p}\left(x^{\prime}\right)\right)^{\prime}=t(T-t)\left(1+c_{1} x^{\varrho}+\frac{c_{2}}{x^{\beta}}\right)\left(1+c_{3}\left|x^{\prime}\right|^{\gamma}+\frac{c_{4}}{\left|x^{\prime}\right|^{\nu}}\right)
$$

where $\phi_{p}(u)=|u|^{p} u$ for $u \in \mathbb{R}, p>-1, c_{j}(1 \leq j \leq 4)$ are positive constants, $\varrho, \gamma, \nu \in$ $(0, \infty)$ and $\beta \in(0,1)$. Also $p>\varrho+\gamma-1$ or $p=\varrho+\gamma-1$ and $p>\left(c_{1} T^{\varrho+3} / 4\right) \max \left\{1, c_{3}, c_{4}\right\}$ -1 . It is clear that $\phi_{p}$ satisfies $\left(\mathrm{H}_{1}\right)$ (with $\phi_{p}$ instead of $\phi$ ). Set

$$
f(t, x, y)=t(T-t)\left(1+c_{1} x^{\varrho}+\frac{c_{2}}{x^{\beta}}\right)\left(1+c_{3}|y|^{\gamma}+\frac{c_{4}}{|y|^{\nu}}\right)
$$

for $(t, x, y) \in[0, T] \times(0, \infty) \times(\mathbb{R} \backslash\{0\})$. Then $f$ satisfies $\left(\mathrm{H}_{2}\right)$ with $\delta(t)=t(T-t)$ and (3.5) with

$$
\begin{array}{ll}
h_{1}(x)=\frac{T^{2}}{4}\left(1+c_{1} x^{\varrho}\right), & h_{2}(x)=\frac{T^{2} c_{2}}{4 x^{\beta}}, \\
\omega_{1}(x)=1+c_{3} x^{\gamma /(p+1)}, & \omega_{2}=\frac{c_{4}}{x^{v /(p+1)}} .
\end{array}
$$

The validity of $\int_{0}^{1} h_{2}(s) d s<\infty$ follows from $\beta \in(0,1)$. For $x \in[1, \infty)$,

$$
\begin{aligned}
G(x) & =\int_{0}^{\phi(x)} \frac{\phi^{-1}(s)}{\omega_{1}(s)+\omega_{2}(s)} d s=\int_{0}^{x^{p+1}} \frac{s^{(1+\gamma) /(p+1)}}{c_{4}+s^{v /(p+1)}\left(1+c_{3} s^{\gamma /(p+1)}\right)} d s \\
& \geq \frac{1}{c} \int_{1}^{x^{p+1}} \frac{s^{(1+v) /(p+1)}}{s^{(\gamma+\gamma) /(p+1)}} d s=\frac{p+1}{c(p+2-\gamma)}\left(x^{p+2-\gamma}-1\right),
\end{aligned}
$$

where $c=\max \left\{1, c_{3}, c_{4}\right\}$ and since

$$
\begin{aligned}
H(x) & =\int_{0}^{x}\left(h_{1}(s)+h_{2}(s)\right) d s=\frac{T^{2}}{4} \int_{0}^{x}\left(1+c_{1} s^{\varrho}+\frac{c_{2}}{s^{\beta}}\right) d s \\
& =\frac{T^{2}}{4}\left(x+\frac{c_{1}}{\varrho+1} x^{\varrho+1}+\frac{c_{2}}{1-\beta} x^{1-\beta}\right)
\end{aligned}
$$

for $x \in[0, \infty)$, we have

$$
\begin{aligned}
\lim _{x \rightarrow \infty} \frac{G(x)}{H(T x)} & \leq \lim _{x \rightarrow \infty} \frac{((p+1) / c(p+2-\gamma))\left(x^{p+2-\gamma}-1\right)}{\left(T^{2} / 4\right)\left(T x+\left(c_{1} /(\varrho+1)\right)(T x)^{\varrho+1}+\left(c_{2} /(1-\beta)\right)(T x)^{1-\beta}\right)} \\
& = \begin{cases}\infty & \text { if } p>\varrho+\gamma-1 \\
\frac{4(p+1)}{c c_{1} T^{+3}} & \text { if } p=\varrho+\gamma-1 \\
0 & \text { if } p<\varrho+\gamma-1 .\end{cases}
\end{aligned}
$$

Applying Theorem 3.10, the BVP (3.46), (3.3) has a solution for each $\alpha \in \mathscr{B}$. 


\section{Application to singular Dirichlet BVPs with sign changing nonlinearities}

4.1. Introduction. In this section we consider the singular Dirichlet boundary value problem

$$
\begin{gathered}
\left(\phi\left(x^{\prime}(t)\right)\right)^{\prime}=f(x(t))-q(t) h(x(t)) x^{\prime}(0)+r(t) p(x(t)) x^{\prime}(T), \\
x(0)=0, \quad x(T)=0
\end{gathered}
$$

where $q, r \in C^{0}[0, T]$ and $f, h, p \in C^{0}(0, \infty)$ are singular at the value 0 in the following sense $\lim _{x \rightarrow 0^{+}} f(x)=-\infty, \lim _{x \rightarrow 0^{+}} h(x)=\infty$ and $\lim _{x \rightarrow 0^{+}} p(x)=\infty$. Notice that (4.1) is the special case of $(1.3)$ with $\left(F_{1} x\right)(t)=x^{\prime}(0)$ and $\left(F_{2} x\right)(t)=x^{\prime}(T)$.

The form of (4.1) is motivated by the regular functional-differential equation

$$
x^{\prime \prime}(t)=f_{1}(t, x(t))-\gamma \alpha_{0} \frac{g_{0}(t, x(t))}{\int_{0}^{1} g_{0}(s, x(s)) d s} x^{\prime}(0)+\gamma \alpha_{1} \frac{g_{1}(t, x(t))}{\int_{0}^{1} g_{1}(s, x(s)) d s} x^{\prime}(1)
$$

considered in [4] together with the Dirichlet boundary conditions $x(0)=0=x(1)$. Under restrictive sign conditions on the continuous functions $f_{1}, g_{0}, g_{1}$ and constants $\gamma, \alpha_{0}, \alpha_{1}$, the existence of a nonnegative solution is proved in [4] by the topological transversality principle. This BVP is a mathematical model for a biological population [4].

We say that $x$ is a positive solution of the BVP (4.1), (4.2) if $x \in C^{1}[0, T], \phi\left(x^{\prime}\right) \in$ $\mathrm{AC}(0, T), x>0$ on $(0, T), x$ satisfies the boundary conditions (4.2) and (4.1) holds on $(0, T)$.

Throughout this section the following assumptions will be used.

$\left(\mathrm{K}_{1}\right) \phi \in C^{0}(\mathbb{R})$ is increasing and odd, $\lim _{x \rightarrow \infty} \phi(x)=\infty$;

$\left(\mathrm{K}_{2}\right)$ There exists a positive constant $K$ and $\gamma \in(1, \infty)$ such that

$$
\Phi(x) \geq K x^{\gamma} \quad \text { for } x \in[0, \infty)
$$

where

$$
\Phi(x)=\int_{0}^{\phi(x)} \phi^{-1}(s) d s \quad \text { for } x \in \mathbb{R}
$$

$\left(\mathrm{K}_{3}\right) f, h, p \in C^{0}(0, \infty)$, where $h, p$ are positive, $f$ vanishes exactly once on $(0, \infty)$, $\lim _{x \rightarrow 0^{+}} f(x)=-\infty, \lim _{x \rightarrow 0^{+}} h(x)=\infty, \lim _{x \rightarrow 0^{+}} p(x)=\infty$ and

$$
\int_{0}^{1} f(x) d x>-\infty, \quad \int_{0}^{1} h(x) d x<\infty, \quad \int_{0}^{1} p(x) d x<\infty ;
$$

$\left(\mathrm{K}_{4}\right) q, r \in C^{0}[0, T]$ are nondecreasing and nonnegative on $[0, T], q(T)>0$ and $r(T)>$ 0 ;

$\left(\mathrm{K}_{5}\right) \liminf _{x \rightarrow \infty}\left(K^{1 /(\gamma-1)} \int_{0}^{x} f(s) d s\right) /\left[\|q\| \int_{0}^{x} h(s) d s+\|r\| \int_{0}^{x} p(s) d s\right]^{\gamma /(\gamma-1)}>1$;

$\left(\mathrm{K}_{6}\right)$ There exists $\Delta>0$ such that $f$ is nondecreasing and $h, p$ are nonincreasing on $(0, \Delta]$.

Remark 4.1. If $\phi$ satisfies $\left(K_{1}\right)$ then the function $\Phi$ defined by (4.5) is even on $\mathbb{R}$ and $\Phi>0$ on $\mathbb{R} \backslash\{0\}$. 
Remark 4.2. Let $\phi$ satisfy $\left(\mathrm{K}_{1}\right)$ and let $\phi(u)-\phi(v) \geq A\left(u^{\beta}-v^{\beta}\right)$ for $u \geq v \geq 0$ where $A$ and $\beta$ are positive constant. Then

$$
\Phi(x)=\int_{0}^{\phi(x)} \phi^{-1}(s) d s \geq \int_{\phi(x / 2)}^{\phi(x)} \phi^{-1}(s) d s \geq \frac{x}{2}\left(\phi(x)-\phi\left(\frac{x}{2}\right)\right) \geq \frac{A}{2}\left(1-\left(\frac{1}{2}\right)^{\beta}\right) x^{\beta+1}
$$

for $x \in[0, \infty)$. Hence (4.4) is satisfied with $K=(A / 2)\left(1-(1 / 2)^{\beta}\right)$ and $\gamma=\beta+1$.

Remark 4.3. If $\left(\mathrm{K}_{1}\right)$ is satisfied and (4.4) is true with a $\gamma \in(0, \infty)$, then $\gamma>1$. To see this notice (4.4) gives

$$
\int_{0}^{x} \phi^{-1}(s) d s \geq K\left(\phi^{-1}(x)\right)^{\gamma}, \quad x \in[0, \infty) .
$$

Hence $\left(\phi^{-1}(x)\right)^{\gamma} \leq(1 / K) \int_{0}^{x} \phi^{-1}(s) d s<(1 / K) \phi^{-1}(x) x$ and

$$
1 \leq \frac{1}{K}\left(\phi^{-1}(x)\right)^{1-\gamma} x
$$

for $x \in(0, \infty)$. Since $\lim _{x \rightarrow 0} \phi^{-1}(x)=0$, we see that $\gamma>1$.

Remark 4.4. From $\left(\mathrm{K}_{4}\right)$ it follows that $q(T)=\|q\|$ and $r(T)=\|r\|$.

4.2. Auxiliary regular BVPs. Let assumption $\left(\mathrm{K}_{3}\right)$ be satisfied. For $n \in \mathbb{N}$, define $f_{n}, h_{n}$, $p_{n} \in C^{0}[0, \infty)$ by the formulas

$$
\begin{aligned}
& f_{n}(x)= \begin{cases}f(x) & \text { if } f(x) \geq-n \\
-n & \text { if } f(x)<-n \text { or } x=0,\end{cases} \\
& h_{n}(x)= \begin{cases}h(x) & \text { if } h(x) \leq n \\
n & \text { if } h(x)>n \text { or } x=0,\end{cases} \\
& p_{n}(x)= \begin{cases}p(x) & \text { if } p(x) \leq n \\
n & \text { if } p(x)>n \text { or } x=0 .\end{cases}
\end{aligned}
$$

Then $($ for $n \in \mathbb{N}$ )

$$
\begin{array}{clrl}
f(x) \leq f_{n}(x), & |f(x)| & \geq\left|f_{n}(x)\right|, \\
h(x) \geq h_{n}(x)>0, & p(x) & \geq p_{n}(x)>0
\end{array}
$$

for $x \in(0, \infty)$. Also $f_{n}(0)=-n$ and $h_{n}(0)=p_{n}(0)=n$ for $n \in \mathbb{N}$. Finally, define continuous functions $f_{n}^{*}, h_{n}^{*}, p_{n}^{*}: \mathbb{R} \rightarrow \mathbb{R}$ by

$$
f_{n}^{*}(x)= \begin{cases}f_{n}(x) & \text { for } x \in[0, \infty) \\ -n-\gamma_{n}|x|^{1 /(\gamma-1)} & \text { for } x \in(-\infty, 0)\end{cases}
$$


where (for $\gamma$ see $\left(\mathrm{K}_{2}\right)$ )

$$
\begin{aligned}
& \gamma_{n}=\frac{2 \gamma}{\gamma-1}\left(\frac{1}{K}\right)^{1 /(\gamma-1)}(n\|q\|)^{\gamma /(\gamma-1)}, \\
& h_{n}^{*}(x)= \begin{cases}h_{n}(x) & \text { for } x \in[0, \infty) \\
n & \text { for } x \in(-\infty, 0),\end{cases} \\
& p_{n}^{*}(x)= \begin{cases}p_{n}(x) & \text { for } x \in[0, \infty) \\
n & \text { for } x \in(-\infty, 0) .\end{cases}
\end{aligned}
$$

Consider the family of regular functional-differential equations

$$
\left(\phi\left(x^{\prime}(t)\right)\right)^{\prime}=\lambda\left(f_{n}^{*}(x(t))-q(t) h_{n}^{*}(x(t)) x^{\prime}(0)+r(t) p_{n}^{*}(x(t)) \min \left\{0, x^{\prime}(T)\right\}\right)
$$

depending on the parameters $\lambda \in[0,1]$ and $n \in \mathbb{N}$.

The following result we will need in the proofs of Lemmas 4.6 and 4.8.

Lemma 4.5. Let assumptions $\left(K_{1}\right)$ and $\left(K_{2}\right)$ be satisfied and let

$$
\Phi(u) \leq|C u|
$$

for a constant $C$ and some $u \in \mathbb{R}$. Then

$$
|u| \leq\left(\frac{|C|}{K}\right)^{1 /(\gamma-1)}
$$

Proof. By Remark 4.1, $\Phi$ is even on $\mathbb{R}$. Hence $\Phi(|u|) \leq|C||u|$ and then from the inequality (see (4.4)) $K|u|^{\gamma} \leq|C||u|$ we get (4.16).

Lemma 4.6. Let assumptions $\left(K_{1}\right)-\left(K_{4}\right)$ be satisfied and let $x(t)$ be a solution of the BVP $(4.14)_{\lambda}^{n},(4.2)$. Then

$$
x(t) \geq 0 \quad \text { for } t \in[0, T] .
$$

Proof. If $\lambda=0$ then $x(t)=a+b t$ for $t \in[0, T]$ with some $a, b \in \mathbb{R}$ and (4.2) yields $a=$ $b=0$. Hence $x=0$ and (4.17) is true. Let $\lambda \in(0,1]$. Suppose that $\min \{x(t): 0 \leq t \leq T\}=$ $x(\xi)<0$. Then (4.2) gives $\xi \in(0, T)$, and consequently $x^{\prime}(\xi)=0$. If $x^{\prime}(0) \geq 0$ then

$$
\left.\left(\phi\left(x^{\prime}(t)\right)\right)^{\prime}\right|_{t=\xi} \leq \lambda f_{n}^{*}(x(\xi))=\lambda\left(-n-\gamma_{n}|x(\xi)|^{1 /(\gamma-1)}\right)<0 .
$$

Hence $\phi\left(x^{\prime}\right)$ is decreasing on a neighbourhood $U$ of the point $t=\xi$ and then $x^{\prime}$ is also decreasing on $U$ since $\phi$ is increasing on $\mathbb{R}$ by $\left(K_{1}\right)$ and as a result $x^{\prime}>0$ on $[0, \xi) \cap U$ and $x^{\prime}<0$ on $(\xi, T] \cap \mathcal{U}$, which contradicts the minimal value of $x$ at $t=\xi$. Therefore $x^{\prime}(0)<0$ and there exists $v \in(0, \xi]$ such that $x^{\prime}(\nu)=0$ and $x^{\prime}<0$ on $[0, v)$. Since

$$
r(t) p_{n}^{*}(x(t)) \min \left\{0, x^{\prime}(T)\right\} x^{\prime}(t) \geq 0, \quad t \in[0, v],
$$


we have

$$
\left(\phi\left(x^{\prime}(t)\right)\right)^{\prime} x^{\prime}(t) \geq \lambda\left(f_{n}^{*}(x(t))-q(t) h_{n}^{*}(x(t)) x^{\prime}(0)\right) x^{\prime}(t)
$$

on $[0, v]$. Integrating $(4.20)$ from 0 to $v$ yields

$$
-\Phi\left(x^{\prime}(0)\right) \geq \lambda\left(\int_{0}^{x(v)} f_{n}^{*}(s) d s-x^{\prime}(0) \int_{0}^{v} q(t) h_{n}^{*}(x(t)) x^{\prime}(t) d t\right)
$$

and using $\int_{0}^{x(v)} f_{n}^{*}(s) d s>0$, we get

$$
\Phi\left(x^{\prime}(0)\right)<x^{\prime}(0) \int_{0}^{v} q(t) h_{n}^{*}(x(t)) x^{\prime}(t) d t .
$$

Now by Lemma 4.5,

$$
\left|x^{\prime}(0)\right| \leq\left(\frac{1}{K}\left|\int_{0}^{\nu} q(t) h_{n}^{*}(x(t)) x^{\prime}(t) d t\right|\right)^{1 /(\gamma-1)} .
$$

From (4.21) and $\Phi(u)>0$ for $u \in \mathbb{R} \backslash\{0\}$ (see Remark 4.1), we also get

$$
\int_{0}^{x(v)} f_{n}^{*}(s) d s<x^{\prime}(0) \int_{0}^{v} q(t) h_{n}^{*}(x(t)) x^{\prime}(t) d t
$$

and using (4.23),

$$
\int_{0}^{x(v)} f_{n}^{*}(s) d s<\left(\frac{1}{K}\right)^{1 /(\gamma-1)}\left(\left|\int_{0}^{v} q(t) h_{n}^{*}(x(t)) x^{\prime}(t) d t\right|\right)^{\gamma /(\gamma-1)} .
$$

Since

$$
\begin{gathered}
\int_{0}^{x(v)} f_{n}^{*}(s) d s=-\int_{0}^{x(\nu)}\left(\gamma_{n}|s|^{1 /(\gamma-1)}+n\right) d s=\gamma_{n}\left(1-\frac{1}{\gamma}\right)|x(\nu)|^{\gamma /(\gamma-1)}+n|x(\nu)|, \\
\left|\int_{0}^{v} q(t) h_{n}^{*}(x(t)) x^{\prime}(t) d t\right| \leq\|q\|\left|\int_{0}^{v} h_{n}^{*}(x(t)) x^{\prime}(t) d t\right|=n\|q\|\left|\int_{0}^{v} x^{\prime}(t) d t\right|=n\|q\||x(\nu)|,
\end{gathered}
$$

we have (see (4.25) and (4.26))

$$
\gamma_{n}\left(1-\frac{1}{\gamma}\right)|x(\nu)|^{\gamma /(\gamma-1)}+n|x(\nu)|<\left(\frac{1}{K}\right)^{1 /(\gamma-1)}(n\|q\||x(\nu)|)^{\gamma /(\gamma-1)} .
$$

Hence

$$
n<\left[\left(\frac{1}{K}\right)^{1 /(\gamma-1)}(n\|q\|)^{\gamma /(\gamma-1)}-\gamma_{n}\left(1-\frac{1}{\gamma}\right)\right]|x(\nu)|^{1 /(\gamma-1)},
$$


contrary to (see (4.13))

$$
\left(\frac{1}{K}\right)^{1 /(\gamma-1)}(n\|q\|)^{\gamma /(\gamma-1)}-\gamma_{n}\left(1-\frac{1}{\gamma}\right)=-\left(\frac{1}{K}\right)^{1 /(\gamma-1)}(n\|q\|)^{\gamma /(\gamma-1)}<0 .
$$

We have proved that (4.17) is true.

Remark 4.7. Lemma 4.6 shows that any solution $x$ of the BVP $(4.14)_{\lambda}^{n},(4.2)$ is nonnegative on $[0, T]$. Hence $x^{\prime}(T) \leq 0$ and the equality

$$
\left(\phi\left(x^{\prime}(t)\right)\right)^{\prime}=\lambda\left(f_{n}(x(t))-q(t) h_{n}(x(t)) x^{\prime}(0)+r(t) p_{n}(x(t)) x^{\prime}(T)\right)
$$

is satisfied on $[0, T]$.

Our next result gives a priori bounds for solutions of the BVP $(4.14)_{\lambda}^{n},(4.2)$.

Lemma 4.8. Let assumptions $\left(K_{1}\right)-\left(K_{5}\right)$ be satisfied. Then there exist positive constants $S_{0}$ and $S_{1}$ independent of $\lambda$ and $n \in \mathbb{N}$ such that

$$
0 \leq x(t)<S_{0}, \quad\left\|x^{\prime}\right\|<S_{1}, \quad t \in[0, T]
$$

for any solution $x$ of the BVP $(4.14)_{\lambda}^{n},(4.2)$.

Proof. Let $x$ be a solution of the BVP $(4.14)_{\lambda}^{n},(4.2)$ for some $\lambda \in[0,1]$ and $n \in \mathbb{N}$. By Lemma 4.6, $x \geq 0$ on $[0, T]$, and therefore $x^{\prime}(0) \geq 0, x^{\prime}(T) \leq 0$ and (see Remark 4.7) the equality $(4.30)$ is satisfied on $[0, T]$. By $\left(K_{5}\right)$, there exists a positive constant $S_{0}$ such that

$$
\int_{0}^{u} f(s) d s>K^{-1 /(\gamma-1)}\left(\|q\| \int_{0}^{u} h(s) d s+\|r\| \int_{0}^{u} p(s) d s\right)^{\gamma /(\gamma-1)}
$$

for any $u \geq S_{0}$, and therefore (see (4.11))

$$
\int_{0}^{u} f_{n}(s) d s>K^{-1 /(\gamma-1)}\left(\|q\| \int_{0}^{u} h_{n}(s) d s+\|r\| \int_{0}^{u} p_{n}(s) d s\right)^{\gamma /(\gamma-1)}
$$

for $u \in\left[S_{0}, \infty\right)$. Let $\max \{x(t): 0 \leq t \leq T\}=x(\xi)>0$. Then from (4.2) it follows that $\xi \in$ $(0, T)$, and consequently $x^{\prime}(\xi)=0$. We now show that $x(\xi)<S_{0}$, that is the first inequality in (4.31) is true. Suppose that $x(\xi) \geq S_{0}$. Then (4.33) yields

$$
\int_{0}^{x(\xi)} f_{n}(s) d s>0
$$

and since $f_{n}$ vanishes exactly once on $[0, \infty)$,

$$
\int_{u}^{x(\xi)} f_{n}(s) d s>0, \quad u \in[0, x(\xi))
$$

Integrating

$$
\left(\phi\left(x^{\prime}(t)\right)\right)^{\prime} x^{\prime}(t)=\lambda\left(f_{n}(x(t))-q(t) h_{n}(x(t)) x^{\prime}(0)+r(t) p_{n}(x(t)) x^{\prime}(T)\right) x^{\prime}(t)
$$


over $[0, \xi]$ and $[\xi, T]$, we get

$$
\begin{aligned}
&-\Phi\left(x^{\prime}(0)\right)=\lambda( \int_{0}^{x(\xi)} f_{n}(s) d s-x^{\prime}(0) \int_{0}^{\xi} q(t) h_{n}(x(t)) x^{\prime}(t) d t \\
&\left.+x^{\prime}(T) \int_{0}^{\xi} r(t) p_{n}(x(t)) x^{\prime}(t) d t\right), \\
& \Phi\left(x^{\prime}(T)\right)=\lambda\left(\int_{x(\xi)}^{0} f_{n}(s) d s-x^{\prime}(0) \int_{\xi}^{T} q(t) h_{n}(x(t)) x^{\prime}(t) d t\right. \\
&\left.+x^{\prime}(T) \int_{\xi}^{T} r(t) p_{n}(x(t)) x^{\prime}(t) d t\right) .
\end{aligned}
$$

By the Bonnet mean value theorem (see, e.g., [7]),

$$
\begin{aligned}
\int_{0}^{\xi} q(t) h_{n}(x(t)) x^{\prime}(t) d t & =q(\xi) \int_{\nu}^{\xi} h_{n}(x(t)) x^{\prime}(t) d t=q(\xi) \int_{x(v)}^{x(\xi)} h_{n}(s) d s \\
& \leq\|q\| \int_{0}^{x(\xi)} h_{n}(s) d s
\end{aligned}
$$

where $\nu \in(0, \xi)$, and similarly

$$
\begin{gathered}
\int_{0}^{\xi} r(t) p_{n}(x(t)) x^{\prime}(t) d t \leq\|r\| \int_{0}^{x(\xi)} p_{n}(s) d s, \\
\int_{\xi}^{T} q(t) h_{n}(x(t)) x^{\prime}(t) d t \geq-\|q\| \int_{0}^{x(\xi)} h_{n}(s) d s, \\
\int_{\xi}^{T} r(t) p_{n}(x(t)) x^{\prime}(t) d t \geq-\|r\| \int_{0}^{x(\xi)} p_{n}(s) d s .
\end{gathered}
$$

Set

$$
A=\|q\| \int_{0}^{x(\xi)} h_{n}(s) d s, \quad B=\|r\| \int_{0}^{x(\xi)} p_{n}(s) d s .
$$

Then (see (4.34), (4.37), (4.39), (4.40))

$$
\Phi\left(x^{\prime}(0)\right)<A x^{\prime}(0)+B\left|x^{\prime}(T)\right|
$$

and (see (4.35), (4.38), (4.41), (4.42) and Remark 4.1)

$$
\Phi\left(\left|x^{\prime}(T)\right|\right)<A x^{\prime}(0)+B\left|x^{\prime}(T)\right| .
$$

If $x^{\prime}(0) \geq\left|x^{\prime}(T)\right|$ then (4.44) gives $\Phi\left(x^{\prime}(0)\right)<(A+B) x^{\prime}(0)$ and from Lemma 4.5 it follows

$$
x^{\prime}(0) \leq\left(\frac{A+B}{K}\right)^{1 /(\gamma-1)} .
$$


Analogously, if $\left|x^{\prime}(T)\right| \geq x^{\prime}(0)$ then (4.45) and Lemma 4.5 yield

$$
\left|x^{\prime}(T)\right| \leq\left(\frac{A+B}{K}\right)^{1 /(\gamma-1)} .
$$

Therefore

$$
\min \left\{x^{\prime}(0),\left|x^{\prime}(T)\right|\right\} \leq\left(\frac{A+B}{K}\right)^{1 /(\gamma-1)} .
$$

Since (see (4.37) with $\lambda \in(0,1],(4.39)$ and (4.40))

$$
\begin{aligned}
\int_{0}^{x(\xi)} f_{n}(s) d s & <x^{\prime}(0) \int_{0}^{\xi} q(t) h_{n}(x(t)) x^{\prime}(t) d t+\left|x^{\prime}(T)\right| \int_{0}^{\xi} r(t) p_{n}(x(t)) x^{\prime}(t) d t \\
& \leq A x^{\prime}(0)+B\left|x^{\prime}(T)\right|
\end{aligned}
$$

we have (see (4.48))

$$
\int_{0}^{x(\xi)} f_{n}(s) d s<\frac{(A+B)^{\gamma /(\gamma-1)}}{K^{1 /(\gamma-1)}},
$$

and then (see (4.33)) $x(\xi)<S_{0}$, contrary to our assumption $x(\xi) \geq S_{0}$. We have verified that $0 \leq x(t) \leq x(\xi)<S_{0}$ for $t \in[0, T]$.

Finally, we prove the second inequality in (4.31). Let $\left\|x^{\prime}\right\|=\left|x^{\prime}(\eta)\right|>0$ for an $\eta \in$ $[0, T]$. By (4.2), $x^{\prime}(\varepsilon)=0$ for some $\varepsilon \in(0, T)$. Assume that $\varepsilon<\eta$ (when $\eta<\varepsilon$ we proceed similarly). Integrating (4.36) over $[\varepsilon, \eta]$ gives

$$
\begin{gathered}
\Phi\left(x^{\prime}(\eta)\right)=\lambda\left(\int_{x(\varepsilon)}^{x(\eta)} f_{n}(s) d s-x^{\prime}(0) \int_{\varepsilon}^{\eta} q(t) h_{n}(x(t)) x^{\prime}(t) d t\right. \\
\left.+x^{\prime}(T) \int_{\varepsilon}^{\eta} r(t) p_{n}(x(t)) x^{\prime}(t) d t\right) .
\end{gathered}
$$

Now using the inequalities

$$
0 \leq x^{\prime}(0) \leq\left|x^{\prime}(\eta)\right|, \quad\left|x^{\prime}(T)\right| \leq\left|x^{\prime}(\eta)\right|
$$

and (see (4.11))

$$
\begin{gathered}
\left|\int_{x(\varepsilon)}^{x(\eta)} f_{n}(s) d s\right| \leq \int_{0}^{s_{0}}\left|f_{n}(s)\right| d s \leq \int_{0}^{s_{0}}|f(s)| d s, \\
\left|\int_{\varepsilon}^{\eta} q(t) h_{n}(x(t)) x^{\prime}(t) d t\right| \leq\|q\| \int_{0}^{s_{0}} h_{n}(s) d s \leq\|q\| \int_{0}^{s_{0}} h(s) d s, \\
\left|\int_{\varepsilon}^{\eta} r(t) p_{n}(x(t)) x^{\prime}(t) d t\right| \leq\|r\| \int_{0}^{s_{0}} p_{n}(s) d s \leq\|r\| \int_{0}^{s_{0}} p(s) d s,
\end{gathered}
$$

we have (see (4.51) and Remark 4.1)

$$
\Phi\left(\left|x^{\prime}(\eta)\right|\right) \leq C_{1}+C_{2}\left|x^{\prime}(\eta)\right|,
$$


where

$$
C_{1}=\int_{0}^{S_{0}}|f(s)| d s, \quad C_{2}=\|q\| \int_{0}^{S_{0}} h(s) d s+\|r\| \int_{0}^{S_{0}} p(s) d s .
$$

Assuming $\left|x^{\prime}(\eta)\right| \geq 1, \Phi\left(\left|x^{\prime}(\eta)\right|\right) \leq\left(C_{1}+C_{2}\right)\left|x^{\prime}(\eta)\right|$ and then

$$
\left|x^{\prime}(\eta)\right| \leq\left(\frac{C_{1}+C_{2}}{K}\right)^{1 /(\gamma-1)}
$$

by Lemma 4.5 . Hence $\left\|x^{\prime}\right\|<S_{1}$ where

$$
S_{1}=\left(\frac{C_{1}+C_{2}}{K}\right)^{1 /(\gamma-1)}+1 .
$$

We now present a result about the solvability of the BVP $(4.14)_{1}^{n},(4.2)$.

Lemma 4.9. Let assumptions $\left(K_{1}\right)-\left(K_{5}\right)$ be satisfied. Then for each $n \in \mathbb{N}$, there exists a solution $x_{n}$ of the BVP $(4.14)_{1}^{n},(4.2)$ and

$$
0 \leq x_{n}(t)<S_{0}, \quad\left\|x_{n}^{\prime}\right\|<S_{1}, \quad t \in[0, T], n \in \mathbb{N},
$$

where $S_{0}$ and $S_{1}$ are positive constants.

Proof. By Lemma 4.8 we know that any solution $x$ of the BVP $(4.14)_{\lambda}^{n}$, (4.2) with $\lambda \in$ $[0,1]$ and $n \in \mathbb{N}$ satisfies (4.31) where $S_{0}$ and $S_{1}$ are positive constants. Let $\alpha, \beta \in C^{1}[0, T] \rightarrow$ $\mathbb{R}$ be defined by

$$
\alpha(x)=x(0), \quad \beta(x)=x(T)
$$

If we prove that for each $\mu \in[0,1]$, all solutions $(A, B) \in \mathbb{R}^{2}$ of the system

$$
\begin{aligned}
& \alpha(A+B t)-\mu \alpha(-A-B t)=0 \\
& \beta(A+B t)-\mu \beta(-A-B t)=0
\end{aligned}
$$

are bounded, then the assertion of our lemma follows from Theorem 2.1 where $(F x)(t)=$ $f_{n}(x(t))-q(t) h_{n}^{*}(x(t)) x^{\prime}(0)+r(t) p_{n}^{*}(x(t)) \min \left\{0, x^{\prime}(T)\right\}$ in (2.2). Since $\alpha(1) \beta(t)-$ $\alpha(t) \beta(1)=T,(A, B)=(0,0)$ is the unique solution of $(4.60)$ for each $\mu \in[0,1]$ (see Remark 2.2), and our proof is finished.

We will need the following result in limiting processes.

Lemma 4.10. Let assumptions $\left(K_{1}\right)-\left(K_{5}\right)$ be satisfied. Then there exists a positive constant c independent of $n$ such that

$$
x(t) \geq \begin{cases}c t & \text { for } t \in\left[0, \frac{T}{2}\right] \\ c(T-t) & \text { for } t \in\left(\frac{T}{2}, T\right]\end{cases}
$$

for any solution $x$ of the BVP $(4.14)_{1}^{n},(4.2)$. 
Proof. Let $x$ be a solution of the BVP $(4.14)_{1}^{n},(4.2)$. Then $x \geq 0$ on $[0, T]$ by Lemma 4.6 and therefore $x^{\prime}(0) \geq 0$ and $x^{\prime}(T) \leq 0$. Thus for $\hat{t}=0$ and $\hat{t}=T$,

$$
\begin{aligned}
\left.\left(\phi\left(x^{\prime}(t)\right)\right)^{\prime}\right|_{t=\hat{t}} & =f_{n}(0)-q(\hat{t}) h_{n}(0) x^{\prime}(0)+r(\hat{t}) p_{n}(0) x^{\prime}(T) \\
& =-n-q(\hat{t}) n x^{\prime}(0)+r(\hat{t}) n x^{\prime}(T) \leq-n,
\end{aligned}
$$

and consequently $x^{\prime}(0)>0$ and $x^{\prime}(T)<0$. With respect to the values of $\left(\phi\left(x^{\prime}\right)\right)^{\prime}$ on $[0, T]$, we will divide the next part of the proof into two cases.

Case 1. Suppose $\left(\phi\left(x^{\prime}\right)\right)^{\prime}<0$ on $[0, T]$. Then $x^{\prime}$ is decreasing and therefore $x$ is concave on $[0, T]$. Also (see $(4.2)) x^{\prime}(\xi)=0$ for a unique $\xi \in(0, T)$. Now we give a positive lower bound for $x(\xi)$. Let

$$
\varepsilon=\max \left\{\left(\phi\left(x^{\prime}(t)\right)\right)^{\prime}: 0 \leq t \leq T\right\}=\left.\left(\phi\left(x^{\prime}(t)\right)\right)^{\prime}\right|_{t=\tau}(<0)
$$

where $\tau \in[0, T]$. Then $x^{\prime}(t) \geq \phi^{-1}(|\varepsilon|(\xi-t))$ for $t \in[0, \xi], x^{\prime}(t) \leq \phi^{-1}(|\varepsilon|(\xi-t))$ for $t \in[\xi, T]$, and consequently

$$
\begin{gathered}
x(\xi)=\int_{0}^{\xi} x^{\prime}(t) d t \geq \int_{0}^{\xi} \phi^{-1}(|\varepsilon|(\xi-t)) d t=\int_{0}^{\xi} \phi^{-1}(|\varepsilon| t) d t \\
-x(\xi)=x(T)-x(\xi)=\int_{\xi}^{T} x^{\prime}(t) d t \leq \int_{\xi}^{T} \phi^{-1}(|\varepsilon|(\xi-t)) d t=-\int_{0}^{T-\xi} \phi^{-1}(|\varepsilon| t) d t .
\end{gathered}
$$

Therefore

$$
x(\xi) \geq \max \left\{\int_{0}^{\xi} \phi^{-1}(|\varepsilon| t) d t, \int_{0}^{T-\xi} \phi^{-1}(|\varepsilon| t) d t\right\} \geq \int_{0}^{T / 2} \phi^{-1}(|\varepsilon| t) d t
$$

If $-\varepsilon=|\varepsilon| \geq 1$, we have $x(\xi) \geq \int_{0}^{T / 2} \phi^{-1}(t) d t$ and then $x$ being concave on $[0, T]$ gives $x(T / 2) \geq(1 / 2) \int_{0}^{T / 2} \phi^{-1}(t) d t$. Let $-\varepsilon<1$. Since $\varepsilon=\left.\left(\phi\left(x^{\prime}(t)\right)\right)^{\prime}\right|_{t=\tau} \leq f_{n}(x(\tau)), f_{n}(x(\tau)) \geq$ $\varepsilon(>-1)$, and therefore

$$
f(x(\tau))=f_{n}(x(\tau)) \geq \varepsilon .
$$

Now $\lim _{u \rightarrow 0^{+}} f(u)=-\infty$ implies $f(u) \leq-1$ for $u \in(0, a]$ with an $a>0$. Then (4.66) gives $x(\tau)>a$ and since $x$ is concave on $[0, T], x(T / 2)>a / 2$. Set $c_{1}=\min \left\{(1 / 2) \int_{0}^{T / 2} \phi^{-1}(t) d t\right.$, $a / 2\}$. Then $c_{1}$ is independent of $n, x(T / 2)>c_{1}$ and

$$
x(t) \geq \begin{cases}\frac{2 c_{1}}{T} t & \text { for } t \in\left[0, \frac{T}{2}\right] \\ \frac{2 c_{1}}{T}(T-t) & \text { for } t \in\left[\frac{T}{2}, T\right] .\end{cases}
$$


Case 2. Suppose $\left.\left(\phi\left(x^{\prime}(t)\right)\right)^{\prime}\right|_{t=\eta}=0$ for an $\eta \in(0, T)$. Set

$$
\begin{aligned}
& a=\min \left\{t: t \in[0, T],\left.\left(\phi\left(x^{\prime}(s)\right)\right)^{\prime}\right|_{s=t}=0\right\}, \\
& b=\max \left\{t: t \in[0, T],\left.\left(\phi\left(x^{\prime}(s)\right)\right)^{\prime}\right|_{s=t}=0\right\} .
\end{aligned}
$$

Then $0<a \leq b<T$ since we know that $\left.\left(\phi\left(x^{\prime}(s)\right)\right)^{\prime}\right|_{t=\bar{t}}<0$ for $\bar{t}=0$ and $\bar{t}=T$. Also denote $\chi \in(0, \infty)$ the unique zero of $f\left(\right.$ see $\left.\left(\mathrm{K}_{3}\right)\right)$. We now show that for any $t \in(0, T)$ satisfying $\left(\phi\left(x^{\prime}(t)\right)\right)^{\prime} \geq 0$

$$
x(t) \geq \chi
$$

Let $\left.\left(\phi\left(x^{\prime}(t)\right)\right)^{\prime}\right|_{t=t_{0}} \geq 0$ for a $t_{0} \in(0, T)$. Since $\left.\left(\phi\left(x^{\prime}(t)\right)\right)^{\prime}\right|_{t=t_{0}} \leq f_{n}\left(x\left(t_{0}\right)\right)=f\left(x\left(t_{0}\right)\right), x\left(t_{0}\right)$ $\geq \chi$ which proves (4.69). Hence $x(a) \geq \chi$ and $x(b) \geq \chi$. From $\left(\phi\left(x^{\prime}\right)\right)^{\prime}<0$ on $[0, a) \cup(b, T]$ we conclude that $x$ is concave on the intervals $[0, a]$ and $[b, T]$, hence

$$
x(t) \geq \begin{cases}\frac{\chi}{a} t & \text { for } t \in[0, a] \\ \frac{\chi}{T-b}(T-t) & \text { for } t \in[b, T] .\end{cases}
$$

Assume $a<b$. If $\min \{x(t): a \leq t \leq b\}=x(\varrho)<\chi$ for a $\varrho \in(a, b)$, then $\left.\left(\phi\left(x^{\prime}(t)\right)\right)^{\prime}\right|_{t=\varrho} \geq$ 0 and (4.69) yields $x(\varrho) \geq \chi$, which is impossible. Therefore $x(t) \geq \chi$ on $[a, b]$. Hence $x(T / 2) \geq \chi / 2$ and

$$
x(t) \geq \begin{cases}\frac{\chi}{T} t & \text { for } t \in\left[0, \frac{T}{2}\right] \\ \frac{\chi}{T}(T-t) & \text { for } t \in\left[\frac{T}{2}, T\right]\end{cases}
$$

which is also true when $a=b$.

Summarizing, (4.67) and (4.71) show that (4.61) is true with

$$
c=\min \left\{\frac{2 c_{1}}{T}, \frac{\chi}{T}\right\}
$$

Clearly, $c$ is independent of $n$.

\subsection{Existence result and an example.}

Theorem 4.11. Suppose that assumptions $\left(K_{1}\right)-\left(K_{6}\right)$ are satisfied. Then the BVP (4.1), (4.2) has a positive solution.

Proof. Let $S_{0}$ and $S_{1}$ be positive constants in Lemma 4.8. Set

$$
\Omega=\left\{x: x \in C^{1}[0, T],\|x\|<S_{0},\left\|x^{\prime}\right\|<S_{1}\right\} .
$$

By Lemmas 4.9 and 4.10 , for each $n \in \mathbb{N}$, there exists a positive solution $x_{n}$ of the BVP 
$(4.14)_{1}^{n},(4.2)$ such that $x_{n} \in \Omega$ and

$$
x_{n}(t) \geq \begin{cases}c t & \text { for } t \in\left[0, \frac{T}{2}\right] \\ c(T-t) & \text { for } t \in\left[\frac{T}{2}, T\right]\end{cases}
$$

where $c$ is a positive constant independent of $n$. Set $t_{*}=\min \left\{\Delta / S_{1}, T / 2\right\}$ where $\Delta$ appears in $\left(\mathrm{K}_{6}\right)$. Then

$$
\begin{gathered}
c t \leq x_{n}(t)=\int_{0}^{t} x_{n}^{\prime}(s) d s \leq S_{1} t \leq \Delta, \quad t \in\left[0, t_{*}\right], \\
c(T-t) \leq x_{n}(t)=-\int_{t}^{T} x_{n}^{\prime}(s) d s \leq S_{1}(T-t) \leq \Delta, \quad t \in\left[T-t_{*}, T\right] .
\end{gathered}
$$

Without loss of generality we can assume that $f(u) \leq 0$ for $u \in(0, \Delta]$. Then (see $(4.11)$ and $\left.\left(\mathrm{K}_{6}\right)\right)$

$$
\begin{gathered}
\left|f_{n}\left(x_{n}(t)\right)\right| \leq\left|f\left(x_{n}(t)\right)\right| \leq|f(c t)|, \quad h_{n}\left(x_{n}(t)\right) \leq h\left(x_{n}(t)\right) \leq h(c t), \\
p_{n}\left(x_{n}(t)\right) \leq p\left(x_{n}(t)\right) \leq p(c t)
\end{gathered}
$$

for $t \in\left(0, t_{*}\right]$, and analogously

$$
\begin{gathered}
\left|f_{n}\left(x_{n}(t)\right)\right| \leq|f(c(T-t))|, \quad h_{n}\left(x_{n}(t)\right) \leq h(c(T-t)), \\
p_{n}\left(x_{n}(t)\right) \leq p(c(T-t))
\end{gathered}
$$

for $t \in\left[T-t_{*}, T\right)$. Set

$$
W_{n}(t)=f_{n}\left(x_{n}(t)\right)-q(t) h_{n}\left(x_{n}(t)\right) x^{\prime}(0)+r(t) p_{n}\left(x_{n}(t)\right) x^{\prime}(T)
$$

for $t \in[0, T], n \in \mathbb{N}$. Then

$$
\begin{gathered}
\left|W_{n}(t)\right| \leq|f(c t)|+S_{1}\|q\| h(c t)+S_{1}\|r\| p(c t), \quad t \in\left(0, t_{*}\right], \\
\left|W_{n}(t)\right| \leq|f(c(T-t))|+S_{1}\|q\| h(c(T-t))+S_{1}\|r\| p(c(T-t))
\end{gathered}
$$

for $t \in\left[T-t_{*}, T\right)$, and

$$
\begin{aligned}
\left|W_{n}(t)\right| \leq & \max \left\{|f(u)|: c t_{*} \leq u \leq S_{0}\right\}+S_{1}\|q\| \max \left\{h(u): c t_{*} \leq u \leq S_{0}\right\} \\
& +\|r\| \max \left\{p(u): c t_{*} \leq u \leq S_{0}\right\}
\end{aligned}
$$

for $t \in\left[t_{*}, T-t_{*}\right]$. Now (4.79)-(4.80) and $\left(\mathrm{K}_{3}\right)$ guarantee the existence a $\varrho \in L_{1}[0, T]$ such that

$$
\left|W_{n}(t)\right| \leq \varrho(t), \quad t \in(0, T), n \in \mathbb{N}
$$


and then, by Theorem 2.3 (statement $(\mathrm{a}))$ with $\left(F_{1} x\right)(t)=x^{\prime}(0)$ and $\left(F_{2} x\right)(t)=x^{\prime}(T)$ in (1.3) and (1.7), there exists a subsequence $\left\{x_{k_{n}}\right\}$ of $\left\{x_{n}\right\}$ converging to $x$ in $C^{1}[0, T]$. Thus $x \in C^{1}[0, T], x$ satisfies the boundary conditions (4.2) and (see (4.74))

$$
x(t) \geq \begin{cases}c t & \text { for } t \in\left[0, \frac{T}{2}\right] \\ c(T-t) & \text { for } t \in\left[\frac{T}{2}, T\right] .\end{cases}
$$

Hence $x>0$ on $(0, T)$ and then

$$
\lim _{n \rightarrow \infty} W_{k_{n}}(t)=f(x(t))-q(t) h(x(t)) x^{\prime}(0)+r(t) p(x(t)) x^{\prime}(T)
$$

for $t \in(0, T)$. By Theorem 2.3 (statement (b)), $x$ is a positive solution of (4.1). We have proved that $x$ is a positive solution of the BVP (4.1), (4.2).

Example 4.12. Consider the differential equation

$$
\left(\left|x^{\prime}\right|^{p-1} x^{\prime}\right)^{\prime}=x^{\alpha}-\frac{1}{x^{\beta}}-t^{3}\left(1+\frac{1}{x^{\varrho}}\right) x^{\prime}(0)+\frac{e^{t}}{x^{\nu}} x^{\prime}(T)
$$

where $p \in(0, \infty), \beta, \varrho, \nu \in(0,1)$ and $\alpha \in(1 / p, \infty)$ or $\alpha=1 / p$ if $\left(p / T^{3}(p+1)\right)^{1+1 / p}>1$. Equation (4.84) is the special case of (4.1) with $\phi(x)=|x|^{p-1} x, f(x)=x^{\alpha}-1 / x^{\beta}, h(x)=$ $1+1 / x^{\varrho}, p(x)=1 / x^{\nu}, q(t)=t^{3}$ and $r(t)=e^{t}$ satisfying assumptions $\left(\mathrm{K}_{1}\right),\left(\mathrm{K}_{3}\right),\left(\mathrm{K}_{4}\right)$ and $\left(\mathrm{K}_{6}\right)$. Since

$$
\Phi(x)=\int_{0}^{\phi(x)} \phi^{-1}(s) d s=\frac{p}{p+1}|x|^{p+1}, \quad x \in \mathbb{R},
$$

$\left(\mathrm{K}_{2}\right)$ is satisfied with $K=p /(p+1)$ and $\gamma=p+1$. From

$$
\begin{aligned}
\lim _{x \rightarrow \infty} \frac{K^{1 /(\gamma-1)} \int_{0}^{x} f(s) d s}{\left[\|q\| \int_{0}^{x} h(s) d s+\|r\| \int_{0}^{x} p(s) d s\right]^{\gamma /(\gamma-1)}} \\
\quad=\lim _{x \rightarrow \infty}\left(\frac{p}{p+1}\right)^{1 / p}\left(\frac{x^{\alpha+1}}{\alpha+1}-\frac{x^{1-\beta}}{1-\beta}\right)\left[T^{3}\left(x+\frac{x^{1-\varrho}}{1-\varrho}\right)+\frac{e^{T}}{1-\nu} x^{1-\nu}\right]^{-1-1 / p} \\
\quad= \begin{cases}\infty & \text { if } \alpha>\frac{1}{p} \\
\left(\frac{p}{T^{3}(p+1)}\right)^{1+1 / p} & \text { if } \alpha=\frac{1}{p}\end{cases}
\end{aligned}
$$

we see that also $\left(\mathrm{K}_{5}\right)$ is satisfied. Applying Theorem 4.11, the BVP (4.84), (4.2) has a positive solution.

\section{Acknowledgment}

This work is supported by Grant no. 201/04/1077 of the Grant Agency of Czech Republic and by the Council of Czech Government MSM 6198959214. 


\section{References}

[1] R. P. Agarwal, D. O'Regan, and S. Staněk, Positive solutions of nonlocal singular boundary value problems, Glasgow Mathematical Journal 46 (2004), no. 3, 537-550.

[2] __ Solvability of singular Dirichlet boundary-value problems with given maximal values for positive solutions, Proceedings of the Edinburgh Mathematical Society. Series II 48 (2005), no. 1, $1-19$.

[3] R. G. Bartle, A Modern Theory of Integration, Graduate Studies in Mathematics, vol. 32, American Mathematical Society, Rhode Island, 2001.

[4] L. E. Bobisud, T. S. Do, and Y. S. Lee, Existence for a diffusing population with returns from the boundary to the interior, Applicable Analysis. An International Journal 72 (1999), no. 1-2, 1-16.

[5] A. Cabada and R. L. Pouso, Existence theory for functional p-Laplacian equations with variable exponents, Nonlinear Analysis. Theory, Methods \& Applications. An International Multidisciplinary Journal. Series A: Theory and Methods 52 (2003), no. 2, 557-572.

[6] K. Deimling, Nonlinear Functional Analysis, Springer, Berlin, 1985.

[7] S. Lang, Real and Functional Analysis, 3rd ed., Graduate Texts in Mathematics, vol. 142, Springer, New York, 1993.

[8] D. O'Regan, Some general existence principles and results for $\left(\phi\left(y^{\prime}\right)\right)^{\prime}=q f\left(t, y, y^{\prime}\right), 0<t<1$, SIAM Journal on Mathematical Analysis 24 (1993), no. 3, 648-668.

[9] I. Rachůnková and S. Staněk, General existence principle for singular BVPs and its application, Georgian Mathematical Journal 11 (2004), no. 3, 549-565.

[10] S. Staněk, A nonlocal boundary value problems with singularities in phase variables, Mathematical and Computer Modelling 40 (2004), no. 1-2, 101-116.

[11] _ A nonlocal singular boundary value problem for second-order differential equations, Miskolc Mathematical Notes. A Publication of the University of Miskolc 5 (2004), no. 1, 91104.

[12]__ Singular nonlocal boundary value problems, Nonlinear Analysis. 63 (2005), 277-287.

Ravi P. Agarwal: Department of Mathematical Sciences, Florida Institute of Technology,

Melbourne, Florida 32901-6975, USA

E-mail address: agarwal@fit.edu

Donal O'Regan: Department of Mathematics, National University of Ireland, Galway, Ireland

E-mail address: donal.oregan@nuigalway.ie

Svatoslav Staněk: Department of Mathematical Analysis, Faculty of Science, Palacký University, Tomkova 40, 77900 Olomouc, Czech Republic

E-mail address: stanek@inf.upol.cz 


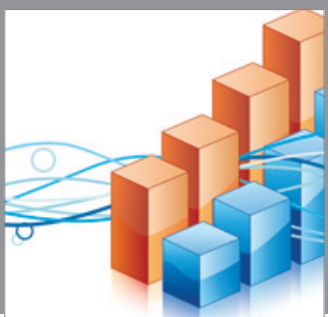

Advances in

Operations Research

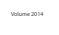

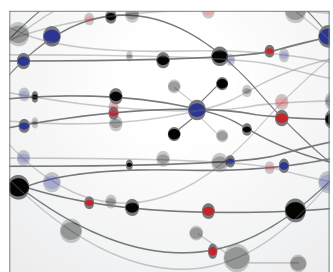

\section{The Scientific} World Journal
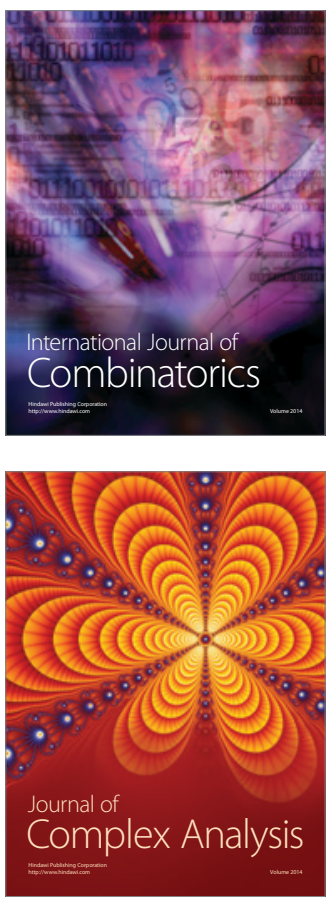

International Journal of

Mathematics and

Mathematical

Sciences
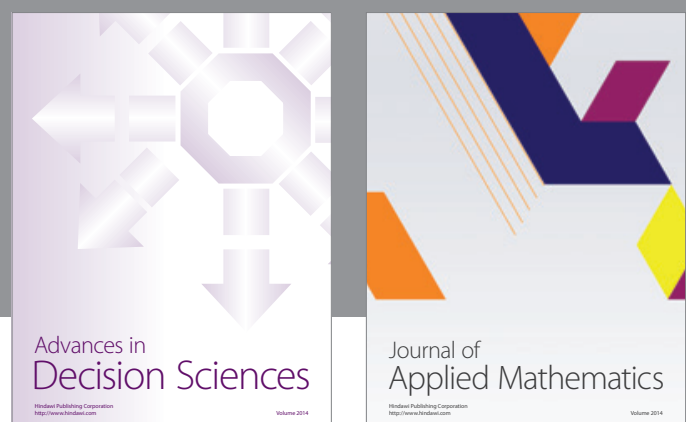

Journal of

Applied Mathematics
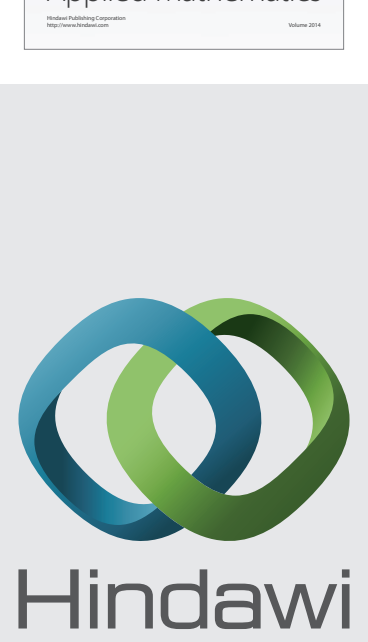

Submit your manuscripts at http://www.hindawi.com
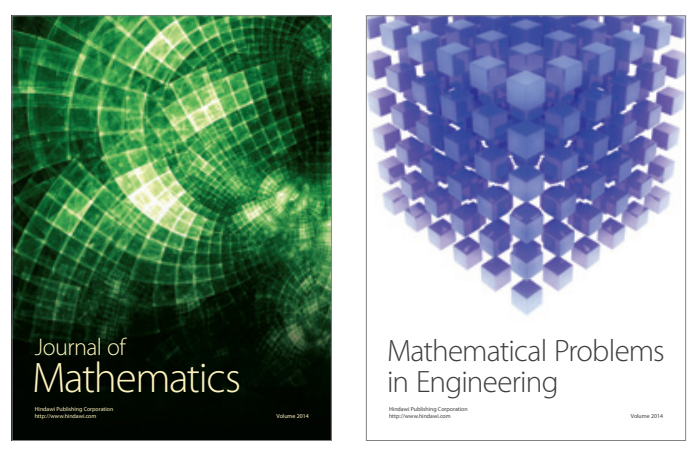

Mathematical Problems in Engineering
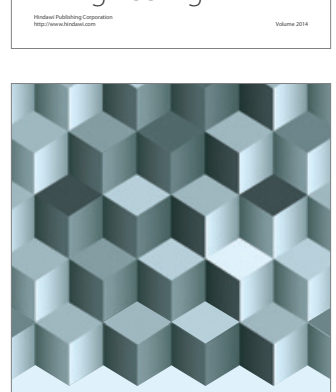

Journal of

Function Spaces
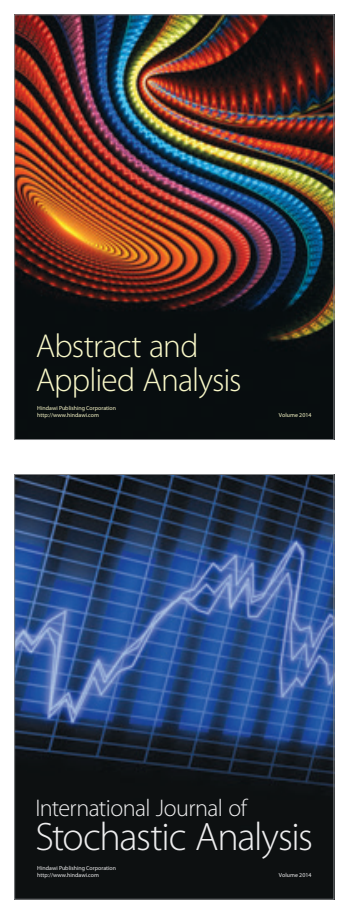

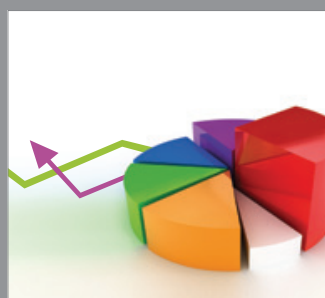

ournal of

Probability and Statistics

Promensencen
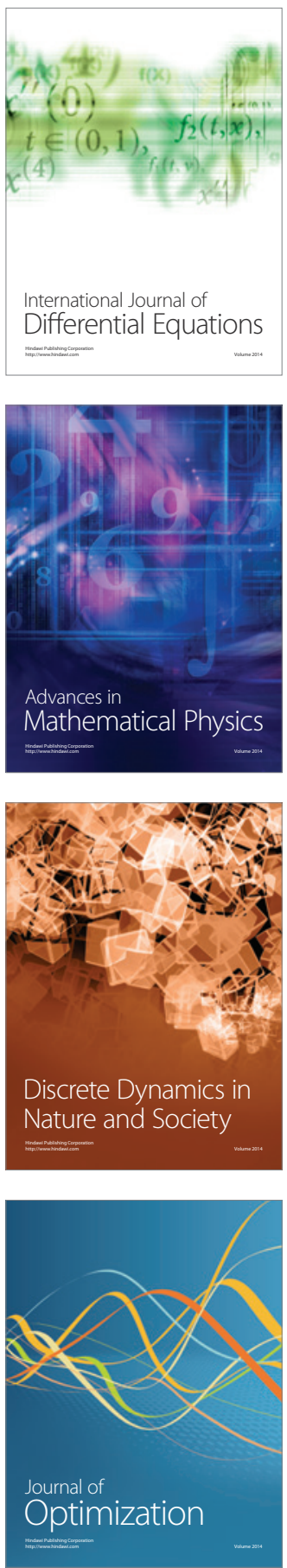